The AstrophysiCAL JouRnaL, 550:824-840, 2001 April 1

(C) 2001. The American Astronomical Society. All rights reserved. Printed in U.S.A.

\title{
THE INVERSE CASCADE AND NONLINEAR ALPHA-EFFECT IN SIMULATIONS OF ISOTROPIC HELICAL HYDROMAGNETIC TURBULENCE
}

\author{
AXel BRANDENBURG ${ }^{1,2}$ \\ Institute for Theoretical Physics, Kohn Hall, University of California, Santa Barbara, Santa Barbara, CA 93106; brandenb@nordita.dk \\ Received 2000 June 12; accepted 2000 December 8
}

\begin{abstract}
A numerical model of isotropic homogeneous turbulence with helical forcing is investigated. The resulting flow, which is essentially the prototype of the $\alpha^{2}$ dynamo of mean field dynamo theory, produces strong dynamo action with an additional large-scale field on the scale of the box (at wavenumber $k=1$; forcing is at $k=5$ ). This large-scale field is nearly force free and exceeds the equipartition value. As the magnetic Reynolds number $R_{m}$ increases, the saturation field strength and the growth rate of the dynamo increase. However, the time it takes to build up the large-scale field from equipartition to its final superequipartition value increases with magnetic Reynolds number. The large-scale field generation can be identified as being due to nonlocal interactions originating from the forcing scale, which is characteristic of the $\alpha$-effect. Both $\alpha$ and turbulent magnetic diffusivity $\eta_{t}$ are determined simultaneously using numerical experiments where the mean field is modified artificially. Both quantities are quenched in an $R_{m}$-dependent fashion. The evolution of the energy of the mean field matches that predicted by an $\alpha^{2}$ dynamo model with similar $\alpha$ and $\eta_{t}$ quenchings. For this model an analytic solution is given that matches the results of the simulations. The simulations are numerically robust in that the shape of the spectrum at large scales is unchanged when changing the resolution from $30^{3}$ to $120^{3}$ mesh points, or when increasing the magnetic Prandtl number (viscosity/magnetic diffusivity) from 1 to 100 . Increasing the forcing wavenumber to 30 (i.e., increasing the scale separation) makes the inverse cascade effect more pronounced, although it remains otherwise qualitatively unchanged.
\end{abstract}

Subject headings: MHD - turbulence

\section{INTRODUCTION}

The generation of large-scale magnetic fields from smallscale turbulence is important in many astrophysical bodies (planets, stars, accretion discs, and galaxies). Over many decades the $\alpha-\omega$ dynamo concept has been invoked to explain large-scale magnetic field generation (Moffatt 1978; Parker 1979; Krause \& Rädler 1980). Over recent years, however, numerical simulations have become available that produce large-scale fields with appreciable magnetic energy, sometimes even exceeding the turbulent kinetic energy (e.g., Glatzmaier \& Roberts 1995; Brandenburg et al. 1995; Ziegler \& Rüdiger 2000). Whether or not large-scale field generation to such amplitudes is related to the $\alpha$-effect remains debatable (e.g., Cattaneo \& Hughes 1996; Brandenburg \& Donner 1997).

The $\alpha$-effect is a key ingredient to many astrophysical dynamo models. The purpose of this paper is, therefore, to study a simple system that is prototypical of the $\alpha$-effect: homogeneous isotropic turbulence that lacks mirror symmetry. Astrophysical dynamos often work in conjunction with shear, i.e., the $\omega$-effect: this case is studied in a second paper (Brandenburg, Bigazzi, \& Subramanian 2000). An isotropic helical turbulent flow is accomplished by adopting a body force corresponding to plane-polarized waves in random directions (but constant polarization) with wavelengths short compared with the size of the box. Since the seminal papers by Frisch et al. (1975) and Pouquet, Frisch, \& Léorat (1976), we know that there should be an inverse

\footnotetext{
${ }^{1}$ Permanent address: Nordita, Blegdamsvej 17, DK-2100 Copenhagen $\varnothing$, Denmark.

${ }_{2}^{2}$ Also: Department of Mathematics, University of Newcastle, Newcastle upon Tyne, NE1 7RU, UK.
}

cascade of magnetic helicity, which has also been demonstrated using direct numerical simulations (e.g., Meneguzzi, Frisch, \& Pouquet 1981; Balsara \& Pouquet 1999). However, to our knowledge there has never been a detailed study of the spatial magnetic field patterns obtained from the inverse cascade, nor has there been a quantitative identification of the classical $\alpha$-effect in mean field dynamo theory. Furthermore, the Reynolds and Prandtl number dependences of this process have not been fully explored yet. In the present paper we study models with strongly helical forcing at different Reynolds numbers. We also investigate some models in which the magnetic Prandtl number (viscosity/magnetic diffusivity) is increased from 1 to 100. This may be important in connection with the Galactic magnetic field, and there are some serious concerns that the inverse cascade may not be efficient at large magnetic Prandtl numbers.

\section{THE MODEL}

We consider a compressible isothermal gas with constant sound speed $c_{s}$, constant dynamical viscosity $\mu$, constant magnetic diffusivity $\eta$, and constant magnetic permeability $\mu_{0}$. The governing equations for density $\rho$, velocity $\boldsymbol{u}$, and magnetic vector potential $\boldsymbol{A}$ are given by

$$
\begin{gathered}
\frac{D \ln \rho}{D t}=-\nabla \cdot \boldsymbol{u}, \\
\frac{D \boldsymbol{u}}{D t}=-c_{s}^{2} \nabla \ln \rho+\frac{\boldsymbol{J} \times \boldsymbol{B}}{\rho}+\frac{\mu}{\rho}\left(\nabla^{2} \boldsymbol{u}+\frac{1}{3} \nabla \nabla \cdot \boldsymbol{u}\right)+\boldsymbol{f}, \\
\frac{\partial \boldsymbol{A}}{\partial t}=\boldsymbol{u} \times \boldsymbol{B}-\eta \mu_{0} \boldsymbol{J},
\end{gathered}
$$


where $\boldsymbol{D} / \boldsymbol{D} t=\partial / \partial t+\boldsymbol{u} \cdot \boldsymbol{\nabla}$ is the advective derivative, $\boldsymbol{B}=\boldsymbol{\nabla} \times \boldsymbol{A}$ is the magnetic field, $\boldsymbol{J}=\boldsymbol{\nabla} \times \boldsymbol{B} / \mu_{0}$ is the current density, and $f$ is a random forcing function.

We use periodic boundary conditions in all three directions for all variables. This implies that the mass in the box is conserved, i.e., $\langle\rho\rangle=\rho_{0}$, where $\rho_{0}$ is the value of the initially uniform density and angular brackets denote volume averages. We adopt a forcing function $f$ of the form

$$
\boldsymbol{f}(\boldsymbol{x}, t)=\operatorname{Re}\left\{N \boldsymbol{f}_{\boldsymbol{k}(t)} \exp [i \boldsymbol{k}(t) \cdot \boldsymbol{x}+i \phi(t)]\right\},
$$

where $\boldsymbol{k}(t)=\left(k_{x}, k_{y}, k_{z}\right)$ is a time-dependent wavevector, $\boldsymbol{x}=(x, y, z)$ is position, and $\phi(t)$ with $|\phi|<\pi$ is a random phase. On dimensional grounds the normalization factor is chosen to be $N=f_{0} c_{s}\left(k c_{s} / \delta t\right)^{1 / 2}$, where $f_{0}$ is a nondimensional factor, $k=|\boldsymbol{k}|$, and $\delta t$ is the length of the time step. We focus on the case in which $|\boldsymbol{k}|$ is around $k_{f}=5$ and select at each time step randomly one of the 350 possible vectors in $4.5<|\boldsymbol{k}|<5.5$. We force the system with eigenfunctions of the curl operator,

$$
f_{k}=\frac{k \times(k \times \hat{e})-i|k|(k \times \hat{e})}{2 k^{2} \sqrt{1-(k \cdot \hat{e})^{2} / k^{2}}},
$$

where $\hat{\boldsymbol{e}}$ is an arbitrary unit vector needed in order to generate a vector $\boldsymbol{k} \times \hat{\boldsymbol{e}}$ that is perpendicular to $\boldsymbol{k}$. Note that $\left|f_{k}\right|^{2}=1$ and, in particular, $i k \times f_{k}=|k| f_{k}$, so the helicity density of this forcing function satisfies

$$
\boldsymbol{f} \cdot \nabla \times f=|\boldsymbol{k}| f^{2}>0
$$

at each point in space. We note that since the forcing function is like a $\delta$-function in $\boldsymbol{k}$-space, this means that all points of $f$ are correlated at any instant in time but are different at the next time step. Thus, the forcing function is $\delta$-correlated in time (but the velocity is not).

We adopt nondimensional quantities by measuring $\boldsymbol{u}$ in units of $c_{s} ; \boldsymbol{x}$ in units of $1 / k_{1}$, where $k_{1}$ is the smallest wavenumber in the box, which has a size of $L=2 \pi$; density in units of $\rho_{0}$; and $\boldsymbol{B}$ in units of $\left(\mu_{0} \rho_{0}\right)^{1 / 2} c_{s}$. This is equivalent to putting

$$
c_{\mathrm{s}}=k_{1}=\rho_{0}=\mu_{0}=1 .
$$

In the following we always quote the mean kinematic viscosity $v \equiv \mu / \rho_{0}$, which is close to the actual kinematic viscosity $\mu / \rho$ because the Mach numbers considered in the present paper are less than 1.

We advance the equations in time using a third-order Runge-Kutta scheme and sixth-order explicit centered derivatives in space. In all cases presented we chose $f_{0}=0.1$, which yields rms Mach numbers around 0.1-0.3 and peak values less than 1 .

Our initial condition is $\ln \rho=\boldsymbol{u}=0$, and $\boldsymbol{A}$ is a smoothed Gaussian random field that is $\delta$-correlated in space, so the initial magnetic energy spectrum is $E_{M}(k) \sim k^{4}$ with a decline at high wavenumbers.

\section{RESULTS}

All the runs are summarized in Table 1. The definitions of various entries to the table are given below, together with an outline of the general behavior of the solutions.

After about 30 time units the rms velocity, $u_{\mathrm{rms}}$, reaches an approximate equilibrium amplitude of up to 0.3. (Since $c_{s}=1$, this is also the Mach number.) This velocity corresponds to a turnover time of $\tau=\ell_{f} / u_{\mathrm{rms}} \approx 4$ time units, where $\ell_{f}=2 \pi / k_{f}$ is the forcing scale. We note that the value of $\tau$ is approximately equal to the value of the correlation time obtained from the temporal correlation function of the velocity. The value of $u_{\mathrm{rms}}$ is somewhat smaller for smaller Reynolds number. The flow has strong positive helicity, as measured by the relative helicity $\langle\boldsymbol{\omega} \cdot \boldsymbol{u}\rangle /\left(\omega_{\mathrm{rms}} u_{\mathrm{rms}}\right)$, which can be as large as $70 \%$ (or even larger when the Reynolds number is smaller). Here $\omega=\nabla \times \boldsymbol{u}$ is the vorticity.

The growth rate of the magnetic field is determined as

\begin{tabular}{|c|c|c|c|c|c|c|c|c|c|}
\hline Parameters & Run 1 & Run 21 & Run 2 & Run 3 & Run $3 p$ & Run 4 & Run 5 & Run 6 & Run 7 \\
\hline Mesh points ....... & $30^{3}$ & $30^{3}$ & $60^{3}$ & $120^{3}$ & $120^{3}$ & $120^{3}$ & $120^{3}$ & $120^{3}$ & $120^{3}$ \\
\hline$v \ldots \ldots \ldots \ldots \ldots \ldots \ldots \ldots \ldots \ldots \ldots \ldots \ldots \ldots \ldots$ & 0.01 & 0.005 & 0.005 & 0.002 & 0.002 & 0.02 & 0.02 & 0.001 & 0.002 \\
\hline$v / \eta \ldots \ldots \ldots \ldots \ldots \ldots \ldots \ldots$ & 1 & 1 & 1 & 1 & 2 & 20 & 100 & 1 & 0.1 \\
\hline$k_{f} \ldots \ldots \ldots \ldots \ldots \ldots \ldots \ldots$ & 5 & 5 & 5 & 5 & 5 & 5 & 5 & 30 & 5 \\
\hline$\left\langle\boldsymbol{u}^{2}\right\rangle_{\operatorname{lin}}^{1 / 2} \ldots \ldots$ & 0.16 & 0.23 & 0.22 & 0.29 & $\ldots$ & 0.11 & 0.114 & 0.082 & 0.29 \\
\hline$\left\langle u^{2}\right\rangle_{\text {sat }}^{1 / 2} \ldots \ldots \ldots \ldots \ldots \ldots$ & 0.12 & 0.15 & 0.15 & 0.18 & 0.19 & 0.10 & 0.104 & 0.062 & 0.20 \\
\hline 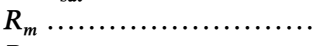 & 80 & 200 & 200 & 600 & 1200 & 700 & 3300 & 400 & 60 \\
\hline$R_{m, \operatorname{lin}}^{m} \ldots \ldots \ldots \ldots \ldots \ldots \ldots \ldots \ldots \ldots$ & 100 & 300 & 300 & 900 & 1800 & 700 & 3600 & 500 & 90 \\
\hline$R_{m, \lambda} \cdots \cdots \cdots \cdots \cdots \cdots \cdots \cdots \cdots \cdots$ & 3 & 9 & 9 & 23 & 46 & 21 & 112 & 3 & 2 \\
\hline$R_{m, \text { forc }}^{m, n} \ldots \ldots \ldots \ldots \ldots \ldots$ & 20 & 60 & 60 & 180 & 360 & 140 & 700 & 17 & 18 \\
\hline$R_{m, \text { forc, crit }} \ldots \ldots \ldots \ldots \ldots \ldots$ & 7.3 & $\ldots$ & 6.9 & 8.9 & 8.9 & 12 & 12 & $\ldots$ & 8.9 \\
\hline$\lambda \ldots \ldots \ldots \ldots \ldots \ldots \ldots \ldots \ldots \ldots \ldots \ldots \ldots \ldots$ & 0.026 & 0.06 & 0.056 & 0.067 & $\ldots$ & 0.03 & 0.04 & 0.075 & 0.036 \\
\hline$\lambda \tau \ldots \ldots \ldots \ldots \ldots \ldots \ldots \ldots$ & 0.24 & 0.32 & 0.34 & 0.30 & $\ldots$ & 0.34 & 0.48 & 0.19 & 0.16 \\
\hline$\left\langle\boldsymbol{B}^{2}\right\rangle_{\text {sat }}^{1 / 2} \ldots \ldots \ldots \ldots \ldots \ldots$ & 0.18 & 0.27 & 0.28 & 0.38 & 0.40 & $>0.2$ & $>0.21$ & $>0.2$ & 0.18 \\
\hline$\left\langle J^{2}\right\rangle_{\text {sat }}^{1 / 2} \ldots \ldots \ldots \ldots \ldots \ldots$ & 0.44 & 0.75 & 0.76 & 1.27 & 1.56 & 0.7 & 1.05 & 1.5 & 0.46 \\
\hline$\left\langle\omega^{2}\right\rangle_{\operatorname{lin}}^{1 / 2} \ldots \ldots \ldots \ldots \ldots \ldots$ & 0.80 & 1.12 & 1.12 & 1.81 & 1.81 & 0.58 & 0.58 & 2.4 & 1.76 \\
\hline$\left\langle\omega^{2}\right\rangle_{\text {sat }}^{1 / 2} \ldots \ldots \ldots \ldots \ldots \ldots$ & 0.65 & 0.78 & 0.82 & 1.23 & 1.38 & 0.55 & 0.55 & 1.8 & 1.03 \\
\hline$\langle\boldsymbol{\omega} \cdot \boldsymbol{u}\rangle_{\operatorname{lin}} \ldots \ldots \ldots \ldots \ldots \ldots$ & 0.13 & 0.24 & 0.24 & 0.37 & 0.37 & 0.063 & 0.063 & 0.20 & 0.36 \\
\hline$\langle\boldsymbol{\omega} \cdot \boldsymbol{u}\rangle_{\mathrm{sat}} \ldots \ldots \ldots \ldots \ldots \ldots$ & 0.08 & 0.10 & 0.11 & 0.16 & 0.17 & 0.058 & 0.055 & 0.10 & 0.19 \\
\hline$\langle\boldsymbol{J} \cdot \boldsymbol{B}\rangle_{\max } \ldots \ldots \ldots \ldots \ldots$ & 0.007 & 0.025 & 0.027 & 0.07 & $>0.03$ & 0.025 & $>0.06$ & 0.040 & 0.006 \\
\hline$\langle\boldsymbol{J} \cdot \boldsymbol{B}\rangle_{\max } /\left(J_{\mathrm{rms}} B_{\mathrm{rms}}\right) \ldots \ldots$ & 0.22 & 0.20 & 0.20 & 0.25 & $\ldots$ & 0.23 & 0.18 & 0.25 & 0.17 \\
\hline$\left|\langle\boldsymbol{J} \cdot \boldsymbol{B}\rangle /\left\langle\boldsymbol{B}^{2}\right\rangle\right|_{\operatorname{lin}} \ldots \ldots \ldots$ & 0.9 & 1.3 & 1.1 & $1-1.5$ & $\cdots$ & 2.2 & 2.4 & 5.5 & 0.70 \\
\hline
\end{tabular}
$\lambda=d \ln \left\langle\boldsymbol{B}^{2}\right\rangle_{\operatorname{lin}}^{1 / 2} / d t$, where the subscript "lin " refers to early

TABLE 1

SUMMARY OF RUNS 
times when the field is still weak on all scales. Ellipses in the table indicate that the run has been restarted from another run, so no data are available for the linear growth phase. Also given is the growth rate normalized with the turnover, $\tau=\ell_{f} /\left\langle\boldsymbol{u}^{2}\right\rangle_{\text {lin }}^{1 / 2}$.

In order to assess the Reynolds number dependence of our results, we have performed three runs, with $v=\eta=0.002$ (run 1), $v=\eta=0.005$ (run 2), and $v=\eta=0.01$ (run 3; see Table 1 for a summary). In order to assess the dependence on magnetic Prandtl number, we have additional runs with $v / \eta=20$ (run 4) and 100 (run 5), as well as one with $v / \eta=0.1$ (run 7). These runs will be explained in detail in $\S 3.5$. In runs $1-5$ and 7 the forcing wavenumber was around 5, but in run 6 we increased it to 30 in order to study the properties of larger scale separation (see $\S 3.5$ ). The rms values of various quantities are reasonably well converged, as can be gauged by comparing run 2 $\left(60^{3}\right.$ mesh points) with run $2 l\left(30^{3}\right.$ mesh points), which has the same values of $\eta$ and $v$. We return to a detailed discussion on the Reynolds number dependence in $\S 3.6$.

In the table we give various magnetic Reynolds numbers: $R_{m}$ is based on the box size $(=2 \pi)$ and the velocity $\left\langle\boldsymbol{u}^{2}\right\rangle_{\text {sat }}^{1 / 2}$ at the time when the magnetic field is saturated, $R_{m, \text { lin }}$ is the same but during the linear growth phase (using $\left\langle\boldsymbol{u}^{2}\right\rangle_{>\text {lin }}^{1 / 2}$ ), $R_{m}, \lambda$ is based on the Taylor microscale $\left\langle\boldsymbol{u}^{2}\right\rangle_{\operatorname{lin}}^{1 / 2} /\left\langle\boldsymbol{\omega}^{2}\right\rangle_{\operatorname{lin}}^{1 / 2}$, and $R_{m \text {,forc }}$ is based on the forcing scale $\ell_{f}$ and $\left\langle\boldsymbol{u}^{2}\right\rangle_{\text {lin }}^{1 / 2}$. The critical values of $R_{m, \text { forc }}$ for the onset of dynamo action are also given and are typically between 7 and 9 . In all cases the onset for dynamo action occurs for $v / \eta<1$, i.e., for magnetic Prandtl numbers less than unity.

The evolution of magnetic and kinetic energies $\left(E_{m}\right.$ and $\left.E_{K}\right)$ is shown in Figure 1 . Note that $E_{K}$ decreases after $E_{m}$ has reached its saturation value. (We note that even after saturation the field continues to grow somewhat, but this will be discussed in full detail in $\S 3$.6.) The relative kinetic helicity changes only slightly before and after saturation. Both the growth rate and the saturation level of the magnetic field increase with increasing Reynolds number and are likely to reach some asymptotic value at sufficiently large Reynolds number.

The level of turbulence may be characterized by the ratio of the turbulent to the microscopic diffusion coefficient for a

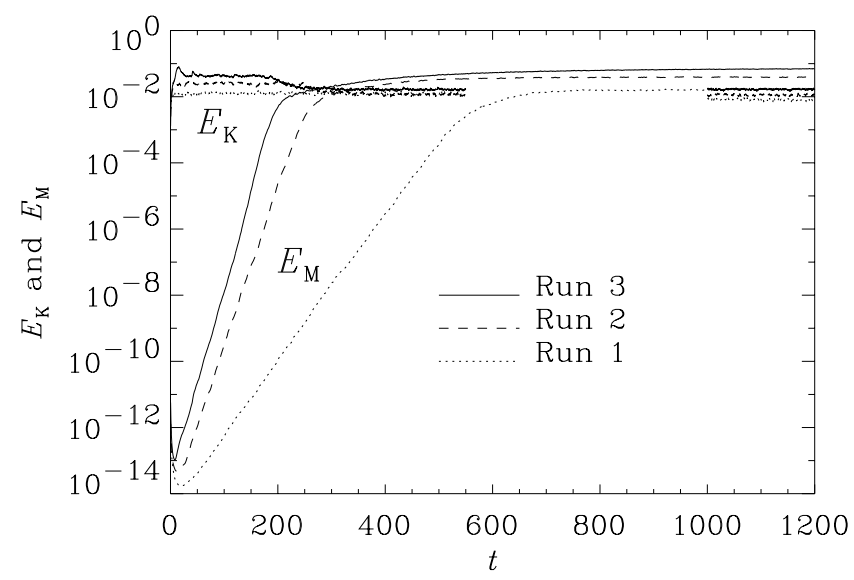

FIG. 1.-Evolution of magnetic energy and kinetic energy (per volume) for three values of the magnetic Reynolds number, $R_{m}$. Note that the growth rate and the saturation level increase with increasing $R_{m}$. For reasons of clarity the curves of kinetic energy are not shown in the range $550<t<1000$. passive scalar, $D_{t} / D$. The standard estimate is $D_{t}=\frac{1}{3} u_{\mathrm{rms}} \ell$, so $D_{t} / D=\frac{1}{3} \mathrm{Re}_{\text {forc }}$. For run 3 we have $\operatorname{Re}_{\text {forc }}=180$, so we expect $D_{t} / D=60$. The actual value obtained by solving the passive scalar advection diffusion equation simultaneously with equations (1)-(3) is somewhat smaller (see $\S 3.4$, where we find $D_{t} / D \approx 40$ for weak fields). This is probably due to the absence of a proper inertial range. Ideally, one would like to simulate higher levels of turbulence, which requires higher resolution. Certain questions therefore cannot be addressed in a satisfactory manner, for example, what the spectral properties of the magnetic field are, especially at large magnetic Prandtl numbers. Addressing this requires the presence of a sufficiently extended inertial range. Other aspects may very well be addressed, for example, what the behavior of the large-scale field is and how it depends on Reynolds and Prandtl numbers. We shall show that the spectral properties are well converged at large scales, but the timescales for reaching a final state increase with magnetic Reynolds number. In order to address these questions, it is important that there is sufficient scale separation between the energy-carrying scale and the scale of the box. Furthermore, it is important to allow for sufficient separation between dynamic and resistive timescales in order to identify properly the mechanisms affecting large-scale dynamo action. A factor of 5 in scale separation seems to be a good compromise, allowing still some degree of turbulent mixing to take place.

\subsection{The Inverse Cascade}

Consistent with previous studies in this field (e.g., Meneguzzi et al. 1981; Balsara \& Pouquet 1999), we find the development of large-scale fields through an inverse cascade effect of the magnetic helicity. This is best seen in the evolution of magnetic energy spectra, $E_{M}(k)$ (see Fig. 2). The kinetic energy spectrum, $E_{K}(k)$, is also shown.

The random initial condition has a $k^{4}$ power spectrum, corresponding to a $\delta$-correlated vector potential. However, even though the initial field was smoothed, the spectrum is deformed significantly during the first few time steps. During the interval $20 \leq t \leq 200$, the spectrum is nearly shape invariant and grows at all scales at the same rate (see Fig. 2). This is typical of small-scale dynamos (Kazantsev 1968).

At $t=200$ the magnetic energy approaches equipartition with the kinetic energy at small scales. After $t=240$ the magnetic energy is in slight superequipartition with the kinetic energy at $k>10$. This marks the beginning of a more complicated process (Fig. 3), during which the field at the largest possible scale $(k=1)$ continues to grow, but the field at intermediate wavenumbers $(k=2,3$, and 4$)$ begins to decline. This process is essentially completed by the time $t=400$. The significance of this process becomes clear when looking at the magnetic field evolution in real space.

\subsection{The Emergence of a Large-Scale Field}

Although the magnetic field reached equipartition already at $t \approx 200$ and its scale began to reach the largest possible scale of the box, it took another 100 time units for the large-scale field at scale $k=1$ to develop fully and, more importantly, to suppress the power at intermediate scales. Looking at $x y$ and $y z$ cross sections, two components of the field $\left(B_{x}\right.$ and $\left.B_{z}\right)$ show the development of a large-scale sinusoidal modulation through the entire box. In Figure 4 we show $x y$ slices of $B_{x}$, but the $y z$ cross sections look 


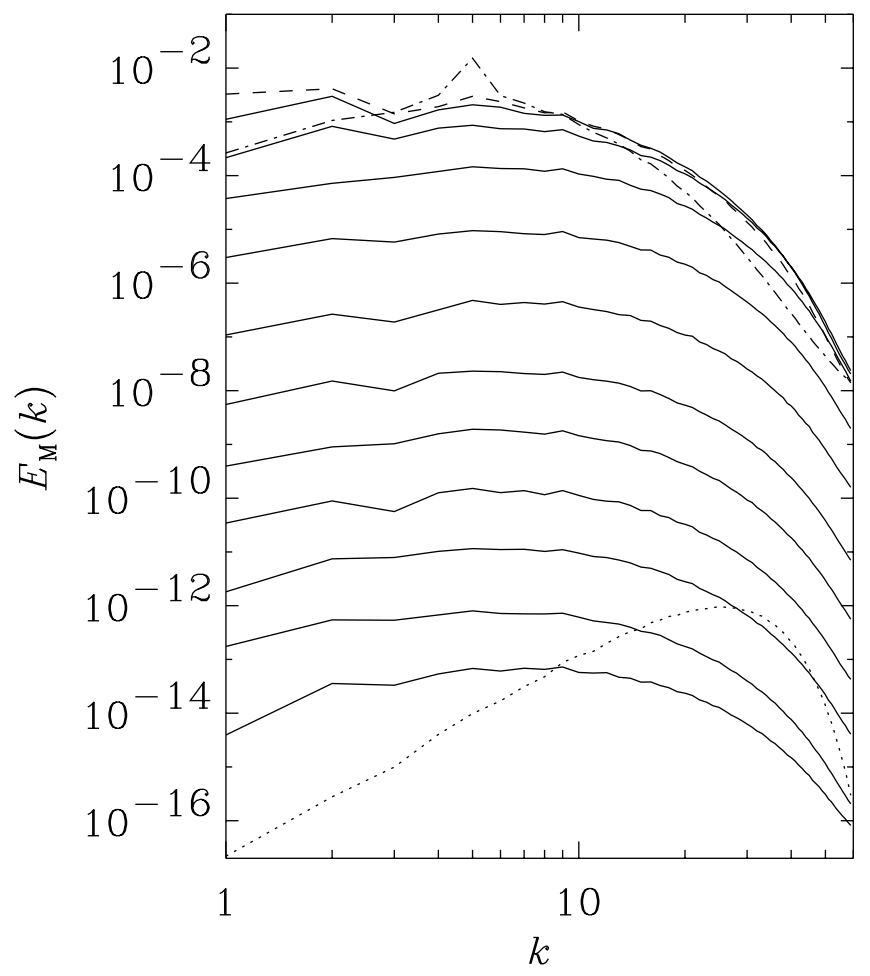

FIG. 2.-Spectra of magnetic energy for run 3 during the initial growth phase at $t=0$ (dotted line); $t=20,40, \ldots, 220$ (solid lines); and $t=240$ (dashed line). The kinetic energy spectrum (time averaged between $600 \leq t \leq 1000$ ) is shown for comparison (the dot-dashed line gives the kinetic energy spectrum).

qualitatively similar, except for a $90^{\circ}$ phase shift of $B_{z}$ in the $y$-direction. This systematic phase shift is seen more clearly in a plot of the three field components averaged in the $x$ and $z$-directions (see Fig. 5).

Although our forcing is isotropic, one particular direction has been selected by the large-scale magnetic field. In runs 1 and 3 it was the $y$-direction, in run 2 the $z$-direction, and in run 5 the $x$-direction. Which direction is selected depends on fine details of the initially random condition. Nevertheless, it is not until the time of saturation that the final selec-

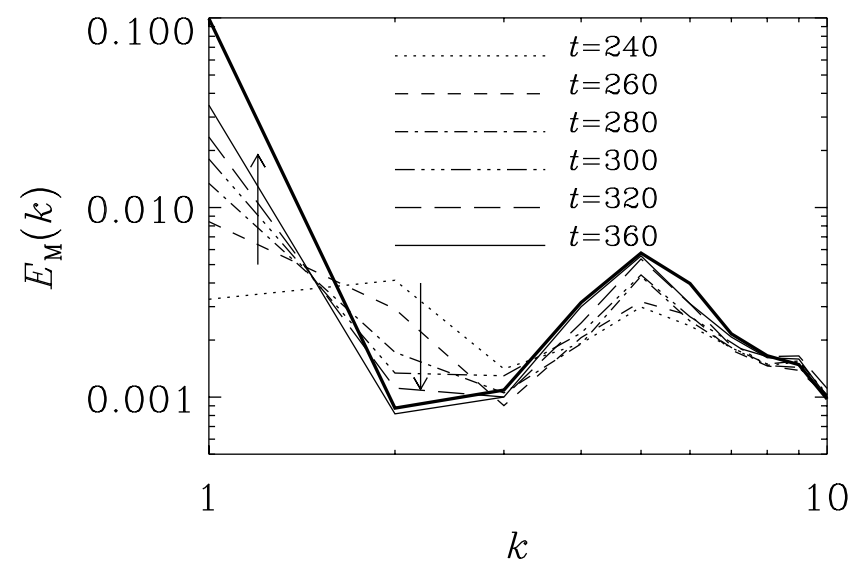

FIG. 3.-Spectra of magnetic energy for run 3 during the saturation phase at times between $t=240$ and 360 . The time-averaged spectrum for times between 600 and 1000 is shown as a thick line. (Only the range $1 \leq k \leq 10$ is shown.) tion is established, as can be seen in Figure 6, where we plot the magnetic energies of the mean field for the three possible directions, denoted by $E\left(K_{x}\right), E\left(K_{y}\right)$, and $E\left(K_{z}\right)$. Hence,

$$
\begin{aligned}
& E\left(K_{x}\right)=\left\langle\langle\boldsymbol{B}\rangle_{y z}^{2}\right\rangle_{x}, \\
& E\left(K_{y}\right)=\left\langle\langle\boldsymbol{B}\rangle_{x z}^{2}\right\rangle_{y}, \\
& E\left(K_{z}\right)=\left\langle\langle\boldsymbol{B}\rangle_{x y}^{2}\right\rangle_{z},
\end{aligned}
$$

where the subscripts denote the directions of averaging. Thus, for any direction $\boldsymbol{K}$, say the $x$-direction, we define corresponding mean fields by averaging in the two perpendicular directions ( $y$ and $z$ in this case), and then we calculate their mean squared value. The time of selection, i.e., when one of the three $E\left(K_{i}\right)$ becomes dominant, is earlier in the large Reynolds number cases.

A quantity of theoretical interest is the ratio $\left\langle\overline{\boldsymbol{B}}^{2}\right\rangle \mid\left\langle\boldsymbol{B}^{2}\right\rangle$, which characterizes the fraction of space occupied by the large-scale field. Initially this ratio is just $\sim 2 \%$ (for run 3 ) and $\sim 0.7 \%$ (for run 5), but later it begins to level off near $80 \%$ (Fig. 7). Most likely, real astrophysical dynamos are far less effective in producing such clean large-scale fields because in reality the helicity of the effective forcing will be far less than $100 \%$. Nevertheless, it is important to notice that it is at least theoretically possible to achieve large-scale field energies near or in excess of the kinetic energy, even though the magnetic Reynolds number is reasonably high.

We note that the phase of the large-scale field may be drifting slowly as long as the large-scale magnetic energy has not yet reached a fully steady state. In run 3, for example, the phase was still drifting slowly in the $y$ direction (speed $\sim 1.5 \eta k_{1}$ ), but then it began to settle after $t \approx 1000$.

\subsection{Spectral Helicity and Energy Transfer}

The primary reason for the large-scale field generation is related to magnetic helicity conservation. Once helicity is injected into the system, it tends to make the magnetic field also helical, as is seen from Figure 8. For a closed or periodic system, however, the net magnetic helicity is conserved, except for diffusion at small scales, i.e.,

$$
\frac{d}{d t}\langle\boldsymbol{A} \cdot \boldsymbol{B}\rangle=-2 \eta\langle\boldsymbol{J} \cdot \boldsymbol{B}\rangle .
$$

Thus, if the magnetic field is to become helical, it must at first have equal amounts of positive and negative helicity. This feature, which is familiar in magnetohydrodynamics (e.g., Seehafer 1996; Ji 1999), is also seen in hydrodynamical simulations (Biferale \& Kerr 1995). At later times, however, magnetic diffusion can destroy magnetic helicity at small scales, leaving magnetic helicity of opposite sign at large scales. This is best described by the evolution equation of the magnetic helicity spectrum, which can be derived from the Fourier transformed induction equation (3),

$$
\frac{\partial \hat{\boldsymbol{A}}_{\boldsymbol{k}}}{\partial t}=\hat{\mathscr{E}}_{\boldsymbol{k}}-\eta \hat{\boldsymbol{J}}_{\boldsymbol{k}}
$$

where hats and subscripts $\boldsymbol{k}$ indicate three-dimensional Fourier transformation and $\mathscr{E}=\boldsymbol{u} \times \boldsymbol{B}$ is the electromotive force. We write down the corresponding equation for the evolution of $\hat{\boldsymbol{B}}_{\boldsymbol{k}}=i \boldsymbol{k} \times \hat{\boldsymbol{A}}_{\boldsymbol{k}}$ and derive from these equations the evolution equation for $\hat{\boldsymbol{A}}_{\boldsymbol{k}} \cdot \hat{\boldsymbol{B}}_{\boldsymbol{k}}^{*}$, where asterisks denote complex conjugation. Note that this is gauge invariant because adding a gradient to $\hat{\boldsymbol{A}}_{\boldsymbol{k}}$ corresponds to adding an 

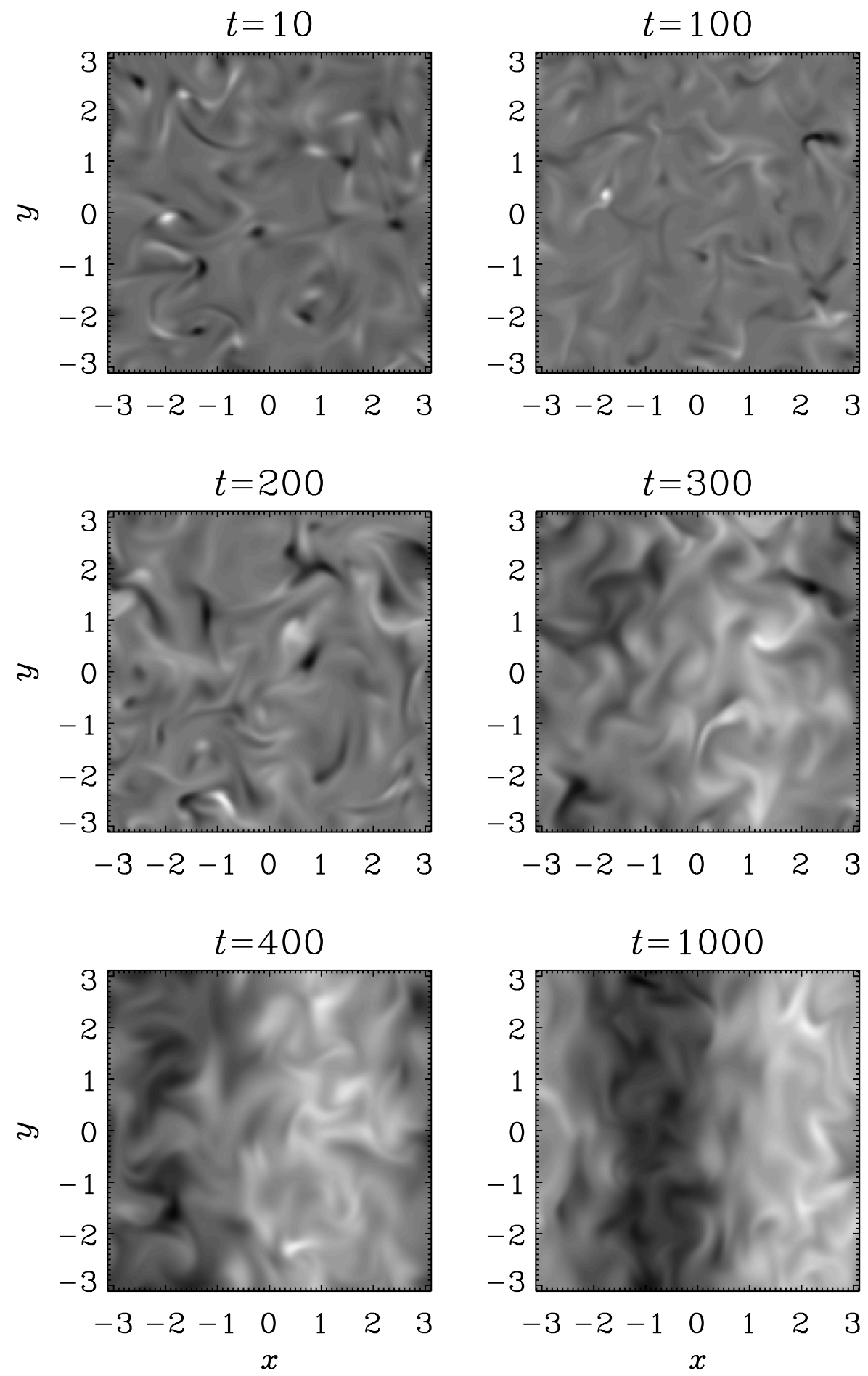

FIG. 4.- Gray-scale images of cross sections of $B_{x}(x, y, 0)$ for run 3 at different times showing the gradual buildup of the large-scale magnetic field after $t=300$. Dark (light) corresponds to negative (positive) values. Each image is scaled with respect to its minimum and maximum values.

$i \boldsymbol{k} \cdot \hat{\boldsymbol{B}}_{\boldsymbol{k}}^{*}$ term that vanishes because the magnetic field is solenoidal. We denote the real parts of the shell-integrated spectra of $\hat{\boldsymbol{A}}_{\boldsymbol{k}} \cdot \hat{\boldsymbol{B}}_{\boldsymbol{k}}^{*}$ and $\hat{\boldsymbol{E}}_{\boldsymbol{k}} \cdot \hat{\boldsymbol{B}}_{\boldsymbol{k}}^{*}$ by $H_{M}(k, t)$ and $S_{M}(k, t)$, respectively, and obtain

$$
\frac{\partial}{\partial t} H_{M}(k, t)=2 S_{M}(k, t)-2 \eta k^{2} H_{M}(k, t) .
$$

Note that $\int H_{M}(k, t) d k=\langle\boldsymbol{A} \cdot \boldsymbol{B}\rangle$ and, because of helicity conservation, $\int S_{M}(k, t) d k=0$, so it makes sense to write equation (13) in the form

$$
\frac{\partial}{\partial t} H_{M}(k, t)=-\frac{\partial}{\partial k} G_{M}(k, t)-2 \eta k^{2} H_{M}(k, t),
$$

where we have defined the spectral flux of helicity,

$$
G_{M}(k, t)=\int_{k}^{\infty} 2 S_{M}\left(k^{\prime}, t\right) d k^{\prime},
$$




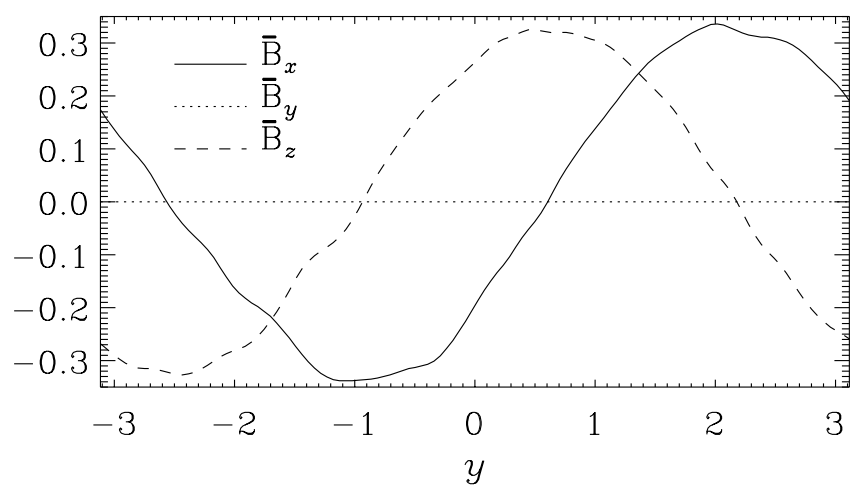

FIG. 5.-Three magnetic field components averaged in the $x$ - and $z$-directions at $t=1000$. Note the $90^{\circ}$ phase shift between $\left\langle\bar{B}_{x}\right\rangle(y)$ and $\left\langle\bar{B}_{z}\right\rangle(y)$ and that the functional form is nearly sinusoidal (run 3 ).

which is plotted in Figure 9 for different times. The magnetic helicity flux, $G_{M}(k, t)$, is found to be always positive, and its peak shifts from small scales $(k \approx 10)$ at early times to large scales $(k=2-3)$ at later times when the magnetic field becomes dynamically important. Positive magnetic helicity is being produced on the right of the maximum of $G_{M}(k, t)$ and negative on the left (see Fig. 10).
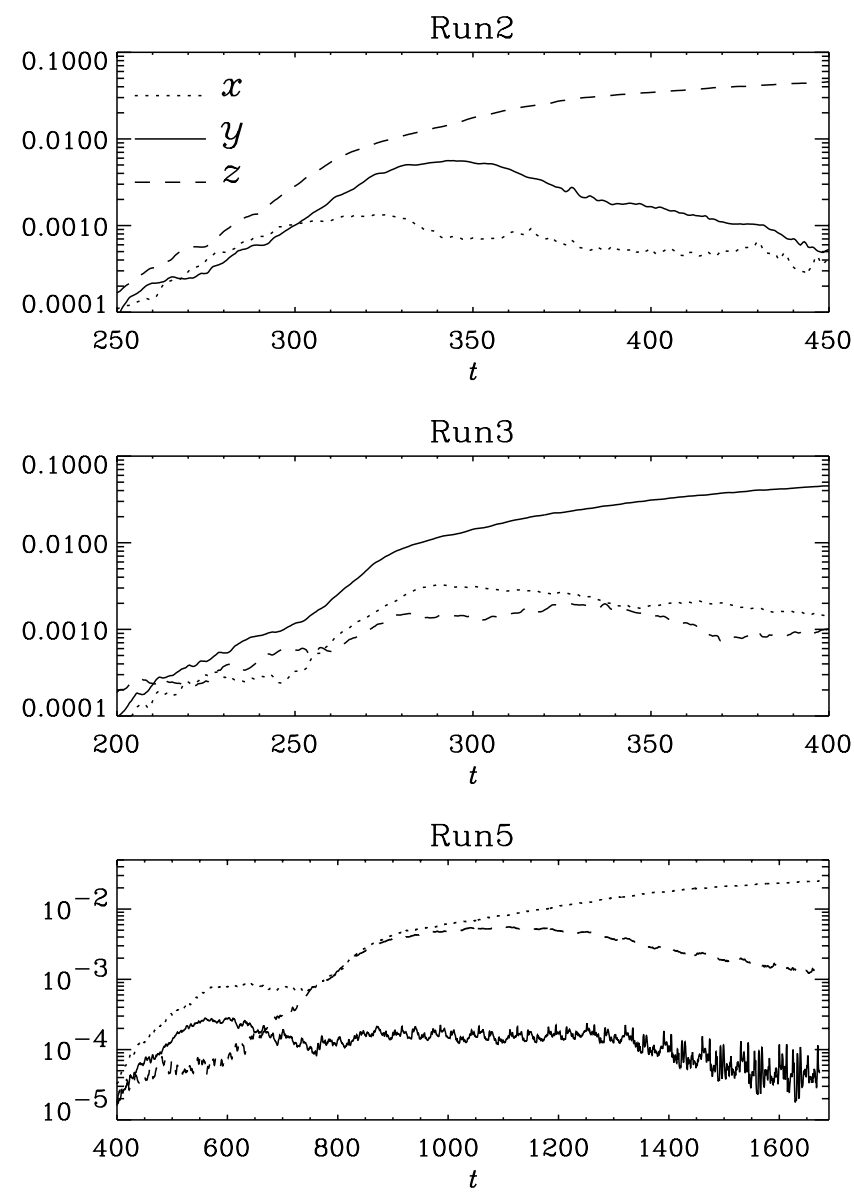

FIG. 6.-Magnetic energies (per volume) of those components of the large-scale field whose wavevectors point in the $x$-, $y$-, or $z$-direction. Which of the three possible force-free solutions is selected in the end depends on chance.

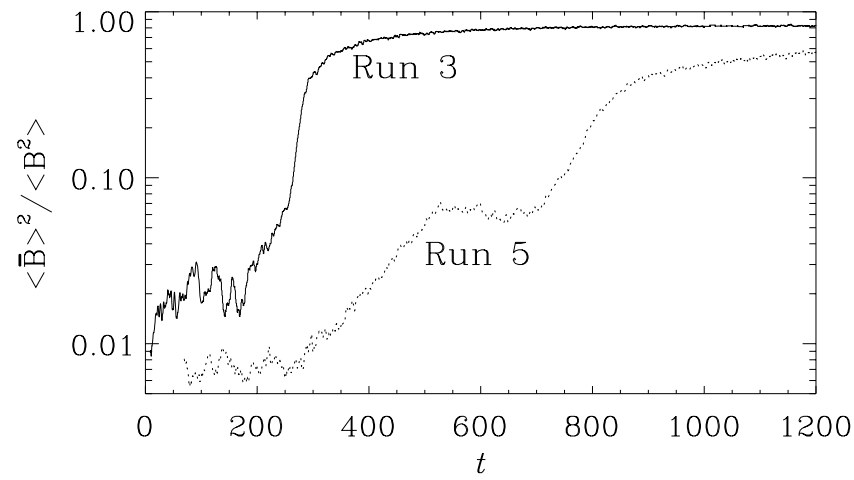

FIG. 7.-Evolution of the ratio $\langle\overline{\boldsymbol{B}}\rangle^{2} /\left\langle\boldsymbol{B}^{2}\right\rangle$, for runs 3 and 5. Note that strong large-scale fields are obtained even for large magnetic Reynolds and Prandtl numbers.

In view of the realizability condition,

$$
E_{M}(k, t) \geq \frac{1}{2} k H_{M}(k, t)
$$

(e.g., Moffatt 1978), the spectral magnetic helicity can be viewed as the driver of spectral magnetic energy: while

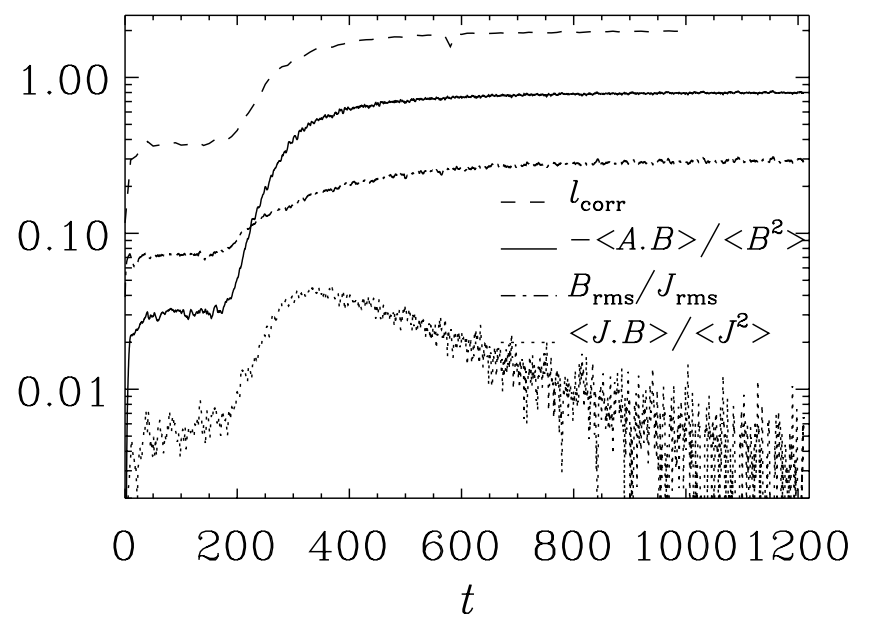

FIG. 8.-Evolution of $-\langle\boldsymbol{A} \cdot \boldsymbol{B}\rangle /\left\langle\boldsymbol{B}^{2}\right\rangle$, compared with other magnetic length scales (run 3).

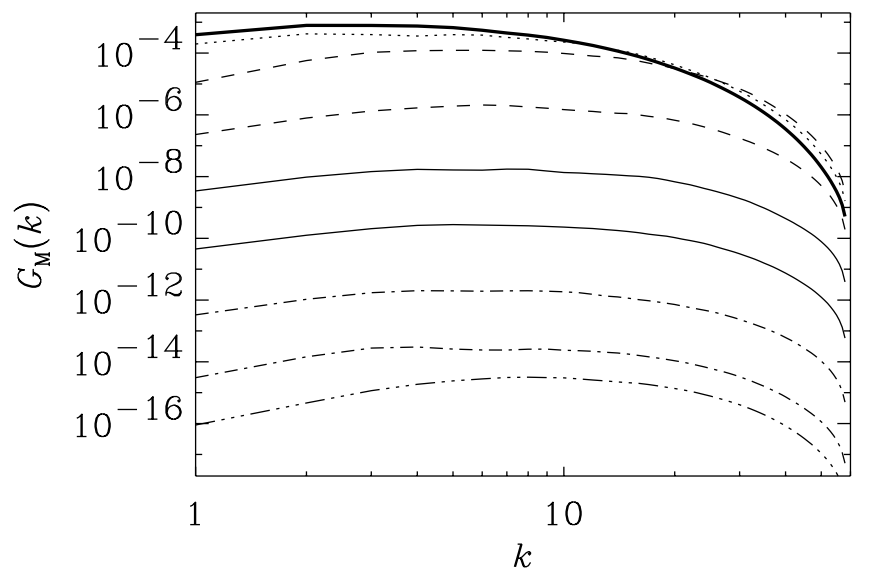

FIG. 9.- Spectra of magnetic helicity flux at $t=10,30$, and 60 (dotdashed line); $t=100$ and 130 (solid lines); $t=160$ and 200 (dashed lines); $t=300$ (dotted line), and for the time average between $t=600$ and 1000 (thick line). 

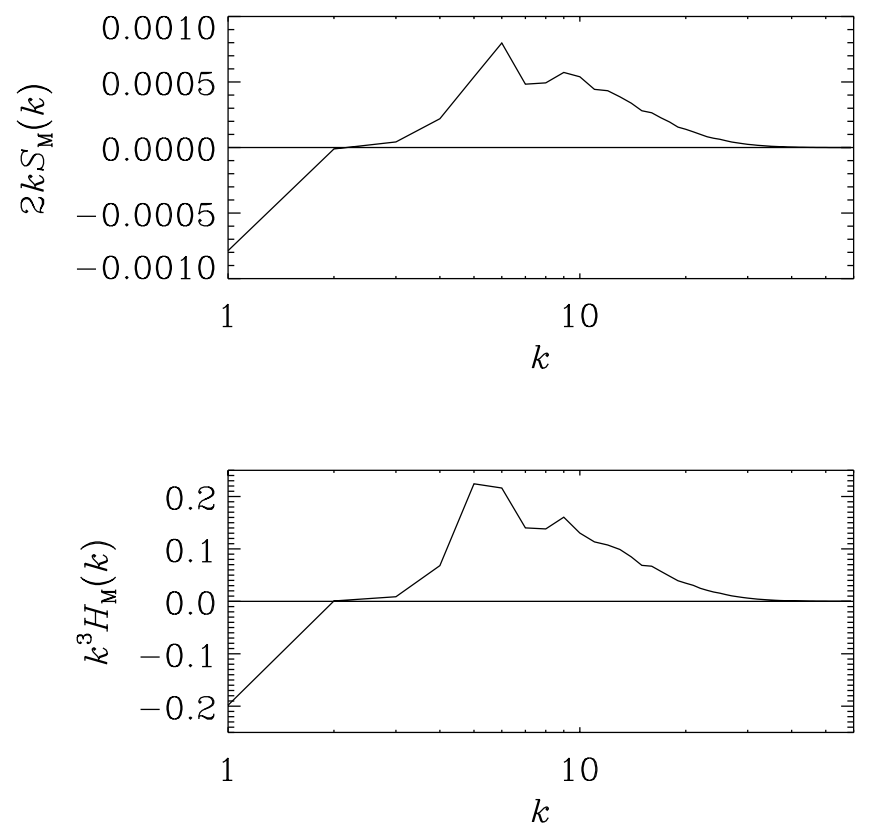

FIG. 10.-Divergence of magnetic helicity flux, $S_{M}(k)$, scaled by $k$, so the areas under the positive and negative parts of the curve are the same when plotted in a lin-log plot. Because of resistive losses of positive magnetic helicity at small scales, the resulting magnetic helicity, $H_{M}(k)$, is now dominated by negative helicity at large scales. The lower plot shows $H_{M}(k)$, scaled by $k^{3}$ to show the contributions from small scales. Both spectra are time averages over the interval $600 \leq t \leq 1000$.

small-scale magnetic helicity is being destroyed, an equal amount gets into the large scales, and this must necessarily enhance the magnetic energy so as to satisfy equation (16). Indeed, in the present simulations the inequality equation (16) is almost saturated at all scales, except at intermediate scales $2 \leq k \leq 4$ (see Fig. 11).

In order to determine the dominant interactions leading to the generation of the large-scale field at $k=1$, we now

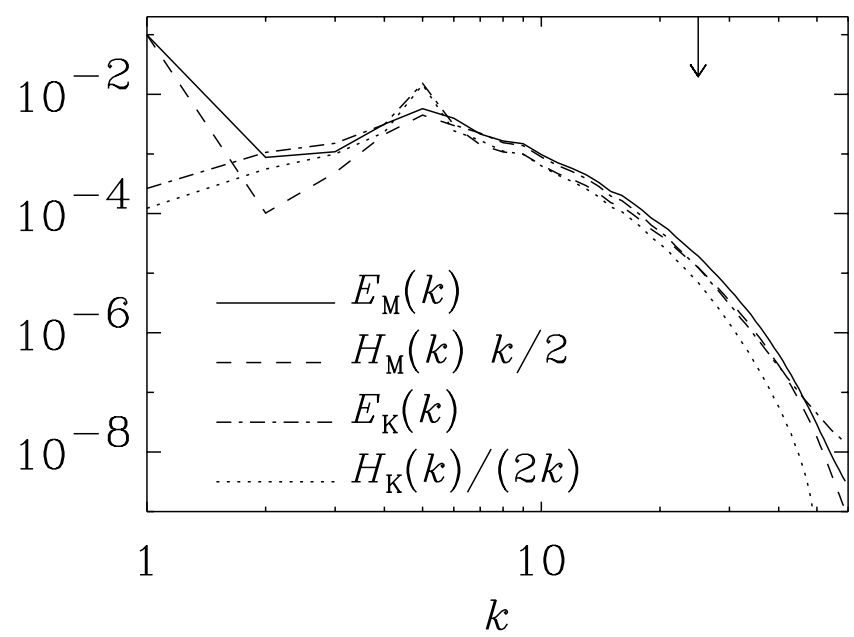

FIG. 11.- Time-averaged spectra of kinetic and magnetic energy, as well as kinetic and magnetic helicity. Note that the magnetic energy exceeds the kinetic energy at $k=1$ and that the inequality (eq. [16]) is almost saturated, except near $k=2$ and 3 . The corresponding realizability condition for the kinetic helicity, on the other hand, is not very sharp. The dissipative cutoff wavenumber, $\left\langle\omega^{2} / v^{2}\right\rangle^{1 / 4}$, is indicated by an arrow at the top. consider the spectral energy equation,

$$
\frac{\partial}{\partial t} E_{M}(k, t)=2 T_{M}(k, t)-2 \eta k^{2} E_{M}(k, t),
$$

where the transfer function of magnetic energy, $T_{M}(k, t)$, is the shell-integrated spectrum of the real part of $\hat{\mathscr{E}}_{\boldsymbol{k}} \cdot \hat{\boldsymbol{J}}_{\boldsymbol{k}}^{*}$. Since $\mathscr{E}=\boldsymbol{u} \times \boldsymbol{B}$, this corresponds really to a triple product,

$$
\sum_{\boldsymbol{k}=\boldsymbol{p}+q}\left(\hat{\boldsymbol{u}}_{\boldsymbol{p}} \times \hat{\boldsymbol{B}}_{q}\right) \cdot \hat{\boldsymbol{J}}_{\boldsymbol{k}}^{*},
$$

where the skew product can also be written as $-\hat{\boldsymbol{u}}_{\boldsymbol{p}} \cdot\left(\hat{\boldsymbol{J}}_{\boldsymbol{k}}^{*} \times \hat{\boldsymbol{B}}_{q}\right)$, emphasizing that this term corresponds to the work done against the Lorentz force. In order to identify the dominant interactions, we have calculated, in real space, the spectral transfer matrix

$$
T_{M}(k, p, q, t)=-\left\langle\boldsymbol{u}_{p} \cdot\left(\boldsymbol{J}_{k} \times \boldsymbol{B}_{q}\right)\right\rangle,
$$

where angular brackets denote volume averages and the subscripts $k, p$, and $q$ denote Fourier filtering around the corresponding wavenumber (by $\pm \frac{1}{2}$ ). (In this notation $\left\langle\boldsymbol{A}_{k} \cdot \boldsymbol{B}_{k}\right\rangle$, for example, is exactly the same as the helicity spectrum.)

In Figure 12 we show $T(k, p)=\sum_{q} T(k, p, q)$, normalized by $\left\langle\boldsymbol{B}^{2}\right\rangle$ for the corresponding times, for $k=1$ and 2 . This function shows that most of the energy of the large-scale field at $k=1$ comes from velocity and magnetic field fluctuations at the forcing scale, $k=5$. At early times this is also true of the energy of the magnetic field at $k=2$, but at late times, $t=1000$, the gain from the forcing scale, $k=5$, has diminished, and instead there is now a net loss of energy into the next larger scale, $k=3$, suggestive of a direct cascade operating at $k=2$ and similarly at $k=3$.

The generation of large-scale energy at $k=1$ through nonlocal inverse energy transfer is characteristic of the $\alpha$ effect in mean field electrodynamics. In the following we shall pursue this analogy further. It should be emphasized, however, that without the simultaneous loss of energy at the next smaller scales (here $k=2$ and 3) through direct energy
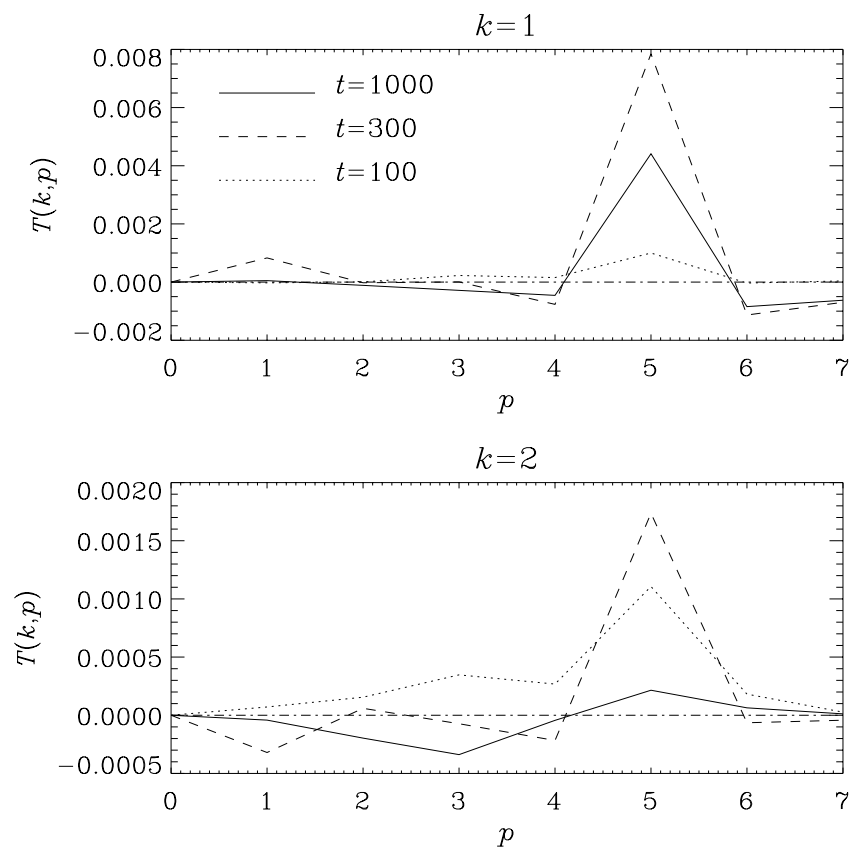

FIG. 12.- Spectral energy transfer function $T(k, p, t)$, normalized by $\left\langle\boldsymbol{B}^{2}\right\rangle$ for three different times, for $k=1$ and 2 (run 3). 
transfer, the $k=1$ field would have been totally swamped by smaller scale fields. Thus, nonlinearity is quite crucial for this process to produce well-defined large-scale fields. Indeed, in the absence of the nonlinear term $\boldsymbol{J} \times \boldsymbol{B}$ in equation (2) the marked large-scale pattern (Fig. 4) disappears within a turnover time. Recent numerical experiments have shown, however, that the ambipolar diffusion nonlinearity also leads to well-defined large-scale fields, even in the absence of the Lorentz force (Brandenburg \& Subramanian 2000).

\subsection{Mean Field Interpretation}

In this subsection we adopt the hypothesis that the largescale component of the field at wavenumber $k=1$ can be described in terms of mean field theory. The magnetic field at other wavenumbers $(k \geq 2)$ is important for contributing to the $\alpha$-effect and the turbulent magnetic diffusivity, $\eta_{t}$, but apart from that it is merely an extra source of noise as far as the dynamics of the large-scale field is concerned. As we have seen in the previous section, this extra noise is automatically kept to a minimum as a result of direct cascade effects and transfer to kinetic energy during the saturation phase.

According to mean field theory for non-mirror symmetric isotropic homogeneous turbulence with no mean flow, the mean magnetic field is governed by the equation

$$
\frac{\partial}{\partial t} \overline{\boldsymbol{B}}=\alpha \boldsymbol{\nabla} \times \overline{\boldsymbol{B}}+\eta_{T} \nabla^{2} \overline{\boldsymbol{B}},
$$

where bars denote the mean fields, $\alpha$ and $\eta_{T}=\eta+\eta_{t}$ are constants, and $\eta_{t}$ is the turbulent magnetic diffusivity. In general, these coefficients are not constant and depend, for example, on the magnetic field. (In our particular case the local magnetic energy density is, however, approximately uniform.) Furthermore, since the magnetic field is strong, $\alpha$ and $\eta_{t}$ should really be replaced by tensors, but we shall ignore this additional modification except that we shall allow $\alpha$ and $\eta_{t}$ to vary slowly in time as the magnetic field approaches saturation. This simplified form of nonlinearity may be justified by noting that the mean magnetic field looks nearly sinusoidal (Fig. 5).

Equation (20) permits steady force-free solutions where the current helicity of the large-scale field, $\overline{\boldsymbol{J}} \cdot \overline{\boldsymbol{B}}$, is given by $\left(\eta_{T} / \alpha\right) \bar{J}^{2}$. Apart from some common phase factor, the mean field depicted in Figure 5 is given by $\overline{\boldsymbol{B}}(y)=(\sin y, 0, \cos y)$, so $\bar{J}=(-\sin y, 0,-\cos y)$, corresponding to negative helicity, and therefore $\alpha$ must be negative. This is in agreement with mean field theory, which predicts that $\alpha$ is a negative multiple of the residual (kinetic minus current) helicity (e.g., Blackman \& Chou 1997; Field, Blackman, \& Chou 1999), which is positive in our case (see Table 1).

If the wavevector of the large-scale field is $K_{y}$ (as in the case discussed above), equation (20) becomes

$$
\begin{gathered}
\dot{\overline{\boldsymbol{B}}}_{x}=-\alpha \overline{\boldsymbol{B}}_{z}^{\prime}+\eta_{T} \dot{\overline{\boldsymbol{B}}}_{x}^{\prime \prime}, \\
\dot{\overline{\boldsymbol{B}}}_{z}=-\alpha \overline{\boldsymbol{B}}_{x}^{\prime}+\eta_{T} \dot{\overline{\boldsymbol{B}}}_{z}^{\prime \prime},
\end{gathered}
$$

where dots and primes denote differentiation with respect to $t$ and $y$, respectively. Since $\alpha<0$, the solution can be written in the form

$$
\begin{aligned}
& \overline{\boldsymbol{B}}_{x}(y, t)=b_{x}(t) \sin (y+\phi), \\
& \overline{\boldsymbol{B}}_{z}(y, t)=b_{z}(t) \cos (y+\phi),
\end{aligned}
$$

where $b_{x}(t)$ and $b_{z}(t)$ are positive functions of time that satisfy

$$
\begin{aligned}
& \dot{b}_{x}=|\alpha| b_{z}-\eta_{T} b_{x}, \\
& \dot{b}_{z}=|\alpha| b_{x}-\eta_{T} b_{z} .
\end{aligned}
$$

In a steady state $|\alpha|=\eta_{T}$ and $b_{x}=b_{z}$. In order to find the actual values of $|\alpha|$ and $\eta_{T}$ during both the saturated steady state and the growth phase, we can do a simple experiment: suppose we put $b_{x}=0$ at some moment in time; then equation (25) would predict that $b_{x}$ starts to recover at the rate $|\alpha| b_{z}$, which allows us to estimate $\alpha$. In practice, we put $b_{x}=0$ by subtracting $\bar{B}_{x}$ from the $x$ component of $B$ at a certain time and restart the simulation with that field.

In order to have a somewhat more precise estimate, we need the solution to equations (25) and (26) for the initial condition $b_{x}(0)=0$ :

$$
\begin{aligned}
& b_{x}=e^{\left(|\alpha|-\eta_{T}\right) t}-e^{-\left(|\alpha|+\eta_{T}\right) t}, \\
& b_{z}=e^{\left(|\alpha|-\eta_{T}\right) t}+e^{-\left(|\alpha|+\eta_{T}\right) t},
\end{aligned}
$$

where the amplitude is arbitrary in linear theory. Adding and subtracting equations (27) and (28), we can solve for $|\alpha|-\eta_{T}$ and $|\alpha|+\eta_{T}$, respectively. In terms of $|\alpha|$ and $\eta_{T}$ separately, we have

$$
\begin{aligned}
& |\alpha|=\frac{1}{2} \frac{d}{d t}\left[\ln \left(b_{x}+b_{z}\right)-\ln \left(-b_{x}+b_{z}\right)\right], \\
& \eta_{T}=\frac{1}{2} \frac{d}{d t}\left[-\ln \left(b_{x}+b_{z}\right)-\ln \left(-b_{x}+b_{z}\right)\right] .
\end{aligned}
$$

In practice, we average the results of equations (29) and (30) over some five to 10 time units. We have applied this method to runs 1-3 at times between $t=100$ and 600 and to run 5 at times between $t=300$ and 1600 (Fig. 13). During these times the mean field was still evolving (Fig. 6), so at different times the mean magnetic field was different, which allows us to obtain the $\alpha(|\boldsymbol{B}|)$ dependence. We take into account the fact that during the experiment the actual field is only $1 / \sqrt{2}$ of what it was before one of the two components of the mean field has been removed. We have then attempted a fit of the form

$$
\alpha=\frac{\alpha_{0}}{1+\alpha_{\mathrm{B}}\left\langle\overline{\boldsymbol{B}}^{2}\right\rangle / B_{\mathrm{eq}}^{2}},
$$

where $B_{\text {eq }}^{2}=\mu_{0} \rho_{0}\left\langle\boldsymbol{u}^{2}\right\rangle_{\text {sat }}$. The result is shown in Figure 14, and the coefficients $\alpha_{0}$ and $\alpha_{B}$ are listed in Table 2. One should note, however, that equation (31) does not accurately represent the actual data of run 5 . Nevertheless, it is clear that $\alpha$ quenching is enhanced for large values of $R_{m}$,

TABLE 2

COEFFICIENTS FOR THE $\alpha$ QUENCHING EXPRESSIONS FOR FOUR Runs WITH DifFERENT VALUES OF $R_{m}$

\begin{tabular}{lcccc}
\hline \hline Parameters & Run 1 & Run 2 & Run 3 & Run 5 \\
\hline$R_{m, \text { lin }} \ldots \ldots$. & 100 & 300 & 900 & 3600 \\
$R_{m, \text { forc }} \ldots \ldots$ & 20 & 60 & 180 & 700 \\
$\left|\alpha_{0}\right| \ldots \ldots \ldots$ & 0.04 & 0.07 & 0.14 & 0.30 \\
$\alpha_{B} \ldots \ldots \ldots$. & 1.4 & 2.4 & 10 & 100 \\
$\left|\alpha_{0}^{\text {imp }}\right| \ldots \ldots$ & 0.040 & 0.076 & 0.092 & 0.035 \\
$\alpha_{B}^{\text {imp }} \ldots \ldots \ldots$ & 1.3 & 4.3 & 14 & 35 \\
\hline
\end{tabular}



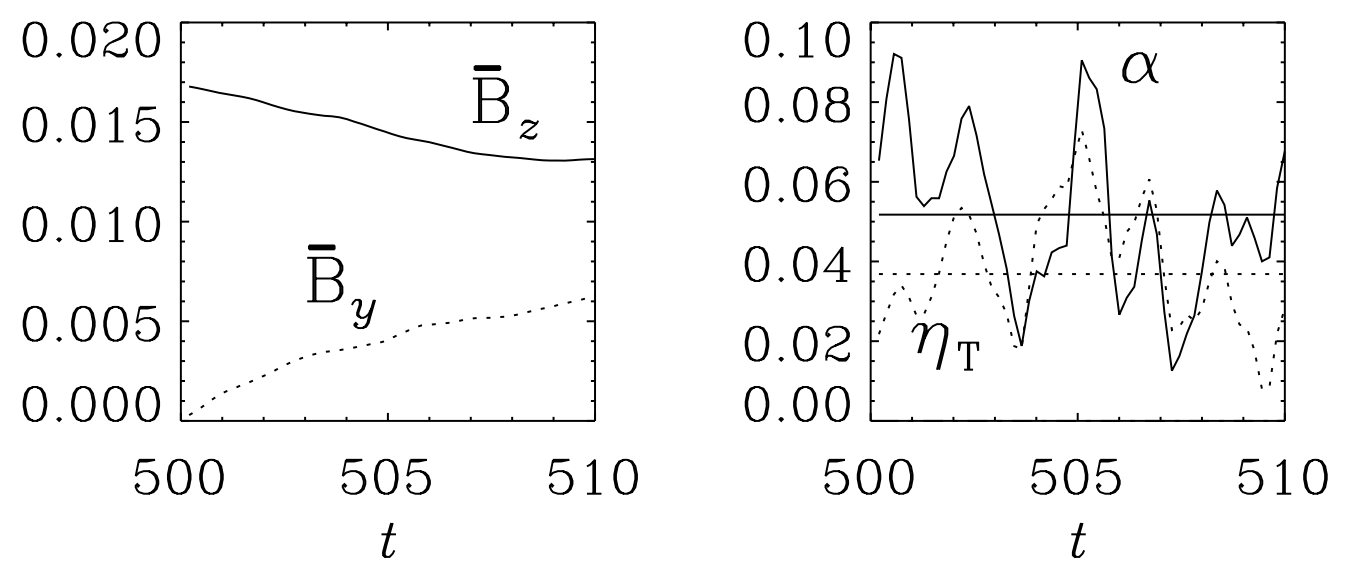

Fig. 13.-Example of the evolution of the mean fields, $\bar{B}_{y}$ and $\bar{B}_{z}$, after subtracting $\bar{B}_{y}$ from the actual field $B_{y}$ (left), and the corresponding results for $\alpha$ and $\eta_{T}($ right). The average values are indicated by horizontal lines (run 5).

which may be described by a fit of the form

$$
\alpha_{B} \approx\left(\frac{R_{m, \text { forc }}}{22}\right)^{1.35}
$$

Such a steep dependence of $\alpha_{B}$ on $R_{m, \text { forc }}$ was suggested by Vainshtein et al. (1993), although his argument (see also Vainshtein \& Cattaneo 1992) assumes the presence of strong small-scale fluctuations (which is not the case here; see Fig. 7).

In Figure 15 we compare with the result for $\alpha$ obtained by just imposing a uniform magnetic field, $B_{0} z$, and calculating $\alpha$ simply as

$$
\alpha^{\mathrm{imp}}=\frac{(\langle\boldsymbol{u} \times \boldsymbol{B}\rangle)_{z}}{B_{0}} .
$$

Each point in Figure 15 corresponds to a different run with given field strength $B_{0}$, but otherwise the same parameters as in runs 1-3 and 5. This method was frequently used in the past (e.g., Brandenburg et al. 1990; Tao, Cattaneo, \& Vainshtein 1993; Cattaneo \& Hughes 1996), but it is not a priori clear that one measures the same $\alpha$ as with the method explained above. Nevertheless, the two results appear to be qualitatively similar (cf. Figs. 14 and 15)

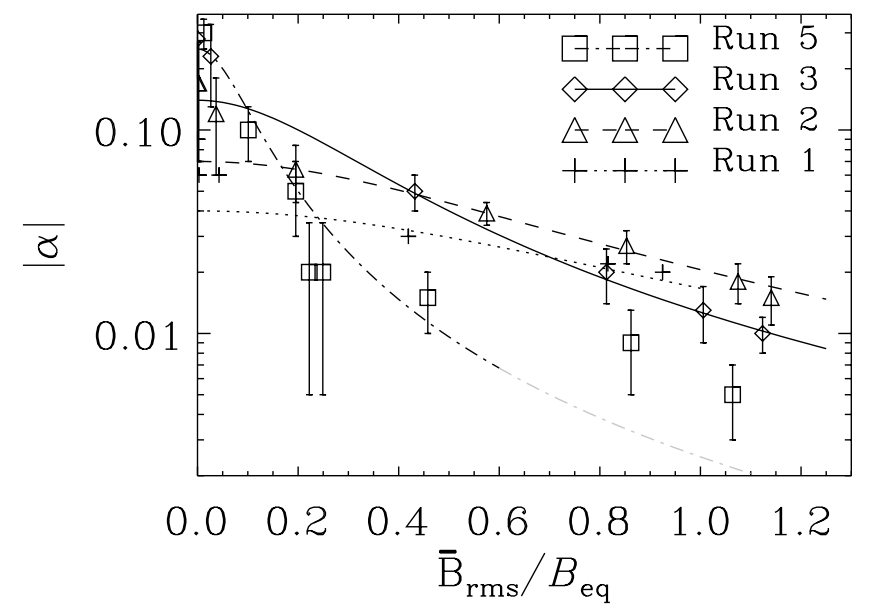

FIG. 14.-Results for $\alpha$ for different values of $R_{m}$ using eq. (29). The lines represent the fits described in the text. although there are some differences in the case in which $R_{m}$ is very large (Table 2). The same values of $\alpha_{B}$ are confirmed by yet another method that is explained below in $\S 3.6$.

The only way a strongly $R_{m}$-dependent $\alpha$ quenching can be compatible with the large-scale field generation seen in the present simulations would be if $\eta_{t}$ was also strongly quenched (see Cattaneo \& Vainshtein 1991 for twodimensional simulations supporting the hypothesis of strong $\eta_{t}$ quenching). In Figure 16 we compare the results obtained for $\eta_{T}$ with the turbulent diffusion coefficient for a passive scalar. The passive scalar diffusion coefficient is obtained by simultaneously solving an additional equation for the concentration per unit mass, $c$,

$$
\frac{D c}{D t}=\frac{1}{\rho} \nabla \cdot \rho D \nabla c,
$$

where $D=\eta$ is chosen for runs $1-3$ and $D=2.5 \eta$ for run 5 . The total (turbulent plus microscopic) passive scalar diffusion coefficient is obtained by measuring the rate at which a narrow Gaussian distribution of $c$ widens as time goes on. The result is shown in the bottom panel of Figure 16. Generally, the suppression of $\eta_{t}$ by magnetic fields is stronger than the suppression of $D_{T}$. $\left(\right.$ For run $3 D_{T} / D$ ranges between

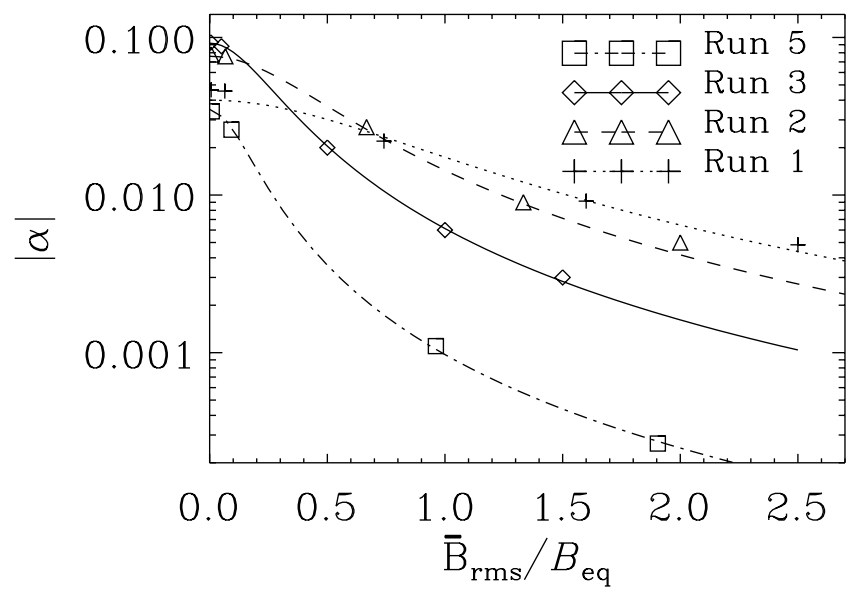

FIG. 15.-Results for $\alpha$ for different values of $R_{m}$ using an imposed magnetic field. The lines represent the fits described in the text. 

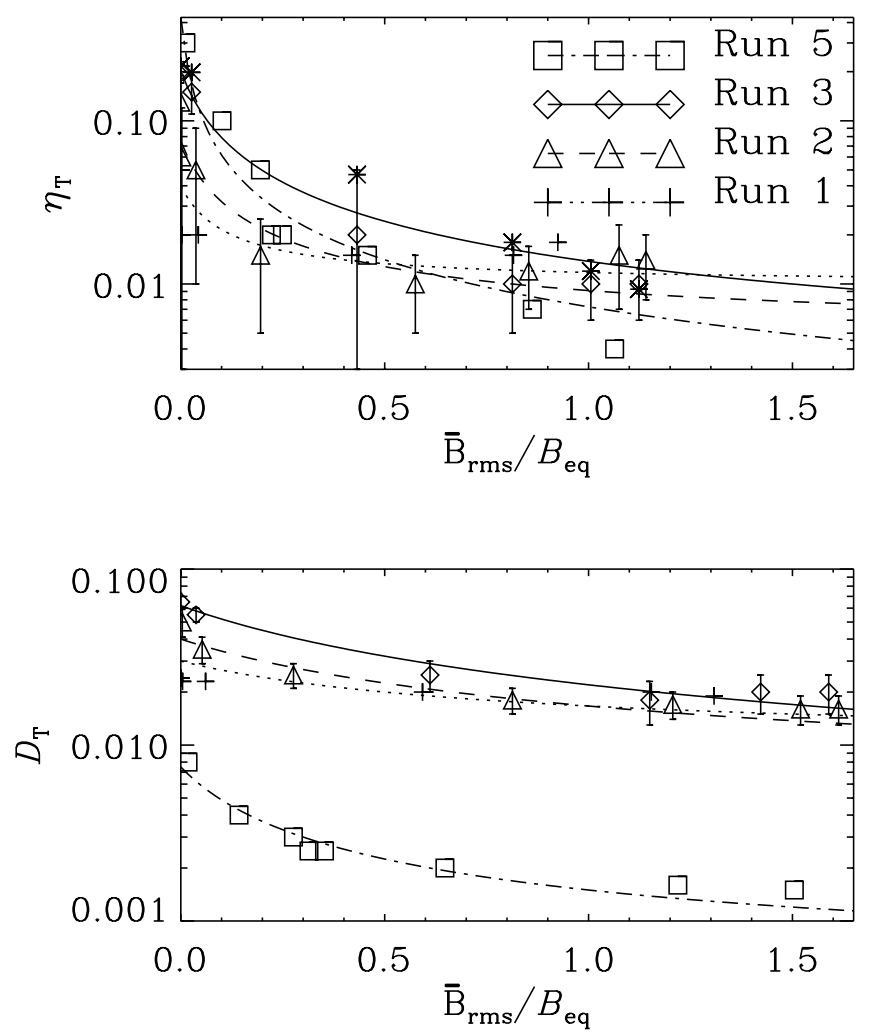

Fig. 16.-Results for $\eta_{T}$ and $D_{T}$ for different values of $R_{m}$. The lines represent the fits described in the text. In the plot of $\eta_{T}$ the asterisks denote $|\alpha|-\lambda$ for the $R_{m, \text { forc }}=120$ run, which agrees reasonably well with $\eta_{T}$.

40 for weak fields and 10 for strong fields.) We note that a dependence of the form

$$
D_{T}=\frac{D+D_{t 0}}{1+D_{B}\langle|\bar{B}|\rangle / B_{\text {eq }}}
$$

seems to fit at least the $D_{T}$ data better than a quadratic dependence. Unlike the results for $\alpha$, the dependence of $D_{B}$ on Reynolds number is here less strong. In the fits shown in Figure 16 a good fit is $D_{B}=2$ for all three runs, and $D_{0}=$ $0.02,0.035$, and 0.06 for runs 1,2 , and 3 , respectively. Run 5 behaves differently because in this large magnetic Prandtl number run there is no inertial range, and so we used $D_{0}=$ 0.007 and $D_{B}=6$.

Determining $\eta_{t}$ from dynamo simulations is notoriously difficult: $\eta_{t}$ has to be determined simultaneously with $\alpha$ from the electromotive force, where $\eta_{t}$ multiplies a derivative of the field, so it is numerically more noisy. Nevertheless, one sees from Figure 16 that $\eta_{t}$ is quenched by more than a factor of 10. However, the functional form cannot be established from our data. Using for $\eta_{T}$ a similar fit formula as equation (35), we have $\eta_{B}=16$ for all three runs; $\eta_{T 0}=$ $0.03,0.07$, and 0.2 for runs 1,2 , and 3 , respectively; and $\eta_{T 0}=0.43, \eta_{B}=60$ for run 5. However, for strong magnetic fields, $\eta_{T}$ levels off at a value of 0.01 (for run 3) or 0.005 (for run 5). These values are similar to the values of $\alpha$. Thus, $\alpha \approx \eta_{T} k_{1}$, which means that the mean field dynamo has turned into a marginally critical state, which is indeed to be expected. As a consistency check for the directly obtained values of $\eta_{T}$, we use the time-dependent growth rate $\lambda(t)$ and show $|\alpha|-\lambda$ (Fig. 16, asterisks) for run 3. They agree reasonably well with $\eta_{T}$ (except near $\langle|\overline{\boldsymbol{B}}|\rangle=0.4 B_{\text {eq }}$ ), suggest- ing that equations (25) and (26) are approximately satisfied with the coefficients obtained above. In $\S 3.6$ we present a more accurate and self-consistent determination of the combined expression for $\alpha$ and $\eta_{t}$ quenching by fitting solutions of equation (20) to the actual evolution of the mean field. Those results support a quadratic quenching formula for both $\alpha$ and $\eta_{t}$.

\subsection{Large-Scale Separation}

In some earlier exploratory models we forced the flow at $k=10$, which gave somewhat more room for the inverse cascade to develop but less room for the direct cascade toward smaller wavenumbers. The latter means that the Reynolds number with respect to the forcing scale is smaller and the turbulent mixing properties, as quantified by the ratio $D_{t} / D$, are poorer. Strong turbulent mixing was important in order to address the issue of Reynolds numberdependent suppression of transport coefficients. In this subsection we shall accept poor mixing in favor of larger scale separation and hence a significantly larger subrange in which the inverse cascade can develop. Thus, we now force the flow at $k=30$ (run 6).

After saturation is reached, which happens first at some intermediate scale, there is a wave of spectral energy propagating toward smaller $k$. This is similar to the results of closure models of Pouquet et al. (1976). However, unlike these models there does not seem to develop a $k^{-1}$ magnetic energy spectrum. Instead, there is only an envelope of the helicity wave that follows approximately a $k^{-1}$ power law (Fig. 17). As before, there is a strong buildup of magnetic energy at the largest scale, $k=1$, combined with a subsequent suppression of energy at intermediate scales $(2 \leq k \leq 20)$. The ratio of the peak value at $k_{1}=1$ to the

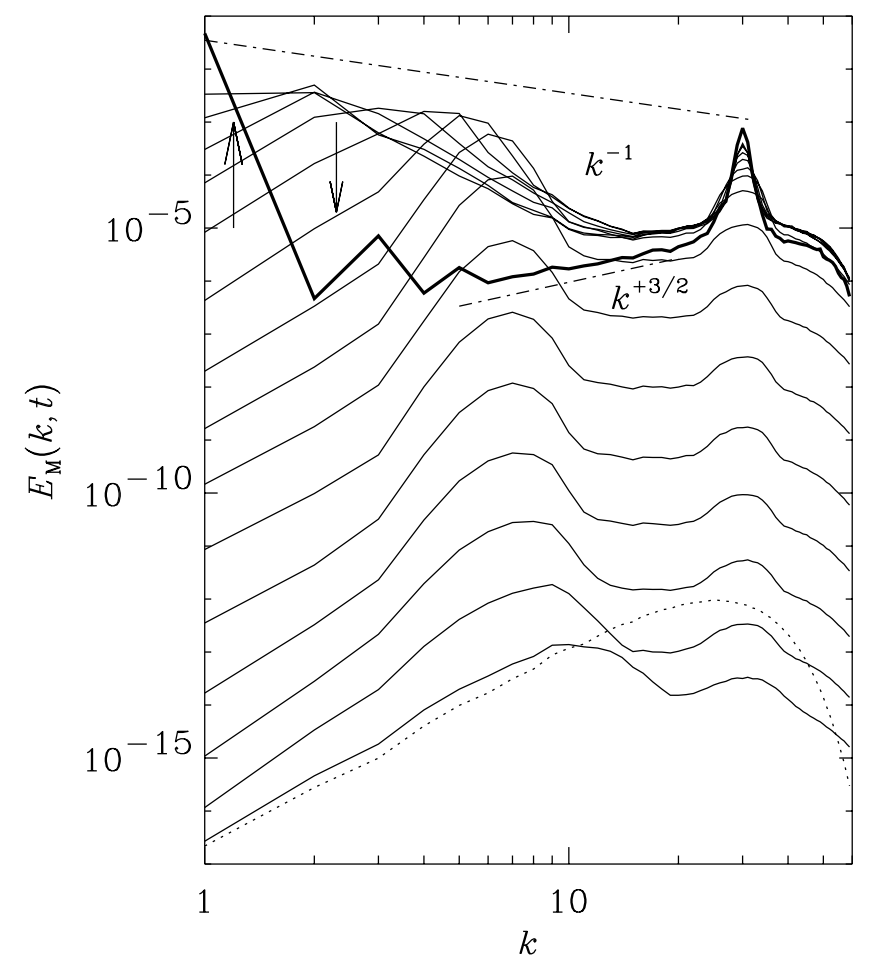

FIG. 17.-Magnetic energy spectra for run 6 with forcing at $k=30$. The times range from 0 (dotted line) to $10,30, \ldots, 290$ (solid lines). The thick solid line gives the final state at $t=1000$. Note that at early times the spectra peak at $k_{\max } \approx 7$. The $k^{-1}$ and $k^{3 / 2}$ slopes are given for orientation as dot-dashed lines. 
value of the secondary peak at the forcing scale $k_{f}=30$ scales like $\left(k_{1} / k_{f}\right)^{-1}$ (Fig. 17). This is also the case in runs with $k_{f}=5$ (Fig. 11).

As a function of time, the spectral magnetic energy grows at the same rate at all values of $k$ until saturation is reached. During early times the magnetic spectra peak at $k_{\max } \approx 7$, which is also the wavenumber that reaches equipartition first, but then the field at this wavenumber decays to a somewhat smaller value while the contributions from the next smaller $k$ grow and subsequently decay (see Fig. 18). Finally, when the energy at $k=1$ reaches equipartition, the energy of all larger values of $k$ becomes suppressed.

Although a comparison with mean field theory may be inappropriate, it is interesting to note that the existence of a $k_{\max }$ is predicted from the theory of $\alpha^{2}$ dynamos in infinite media (Moffatt 1978). The dispersion relation is

$$
\lambda=|\alpha| k-\eta_{T} k^{2},
$$

where $\lambda$ is the growth rate. Maximum growth occurs at wavenumber

$$
k_{\max }=\frac{1}{2} \frac{|\alpha|}{\eta_{T}},
$$

and there the maximum growth rate is

$$
\lambda_{\max }=\frac{1}{2}|\alpha| k_{\max } .
$$

Since $k_{\max }$ and $\lambda_{\max }$ can be measured, we can determine

$$
|\alpha|=\frac{2 \lambda_{\max }}{\mid k_{\max }}, \quad \eta_{T}=\frac{\lambda_{\max }}{\mid k_{\max }^{2}}
$$

during the growth phase of the dynamo. With $k_{\max }=7$ (Fig. 18) and $\lambda_{\text {max }}=0.07$ (Fig. 17) we have $|\alpha|=0.02$ and $\eta_{T}=0.0014$. In this case, the forcing occurs essentially in the dissipation range, so the turbulence therefore has poor mixing properties $\left(\eta_{T} / \eta=1.4\right)$. The value of $\alpha$ is about 7 times smaller than that of run 3 during the kinematic regime, which seems reasonable.

In the nonlinear regime there are marked differences between mean field theory and simulations. Using a nonlinear mean field model of the inverse cascade with $\alpha$-effect, Galanti, Sulem, \& Gilbert (1991) found significant power at $k \geq 2$, which is in contrast to the behavior seen in Figure 17. Consequently, the mean fields of Galanti, Gilbert, \& Sulem (1990) look much less sinusoidal than in the simulations (Fig. 5). If one wanted to model this within the framework of

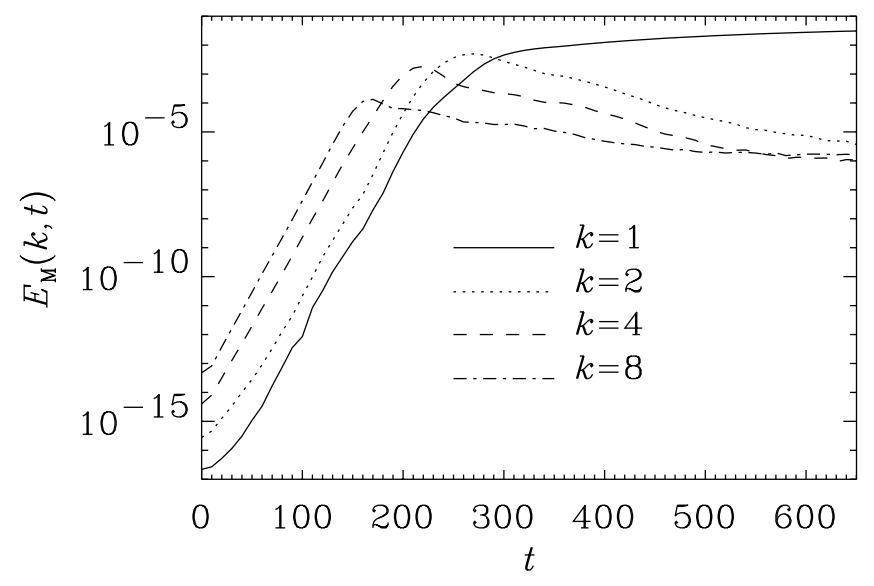

FIG. 18.-Evolution of spectral magnetic energy for selected wavenumbers in a simulation with forcing at $k=30$. an $\alpha$-effect, one would need to invoke a scale-dependent $\alpha$ integral kernel, where the dominant contributions to $\alpha$ come only from the largest scale of the system (Brandenburg \& Sokoloff 2000).

\subsection{Reynolds Number Dependence and Magnetic Helicity}

In this section we first discuss to what extent our results are affected by the limited resolution and finite magnetic Reynolds number. We then show that magnetic helicity conservation implies slow saturation of helical magnetic fields and that this feature is quantitatively reproduced by a mean field model with $R_{m}$-dependent quenching of $\alpha$ and $\eta_{t}$.

In Figure 19 we show energy spectra of the magnetic field for three values of $R_{m}$. The three curves differ by a certain factor but are otherwise essentially the same at small wavenumbers. The main difference is, as expected, at the smaller scales: the spectra for large $R_{m}$ begin to show signs of an inertial range for values of $k$ larger than the forcing wavenumber.

Although the magnetic energy spectra of the statistically steady state seem to show convergence to a spectrum roughly compatible with $k^{-5 / 3}$, there are some serious concerns about the timescale on which such a steady state is achieved. As was first pointed out by Berger (1984), in a closed box (periodic or perfectly conducting) there is an upper bound on the rate of change of the magnetic helicity. This is relevant because the fields that are generated in the simulations have strong magnetic helicity: $\langle\boldsymbol{A} \cdot \boldsymbol{B}\rangle /\left\langle\boldsymbol{B}^{2}\right\rangle \approx-0.8 / k_{1}$ (Fig. 8). Most of the magnetic helicity is in the large scales (Fig. 11). In order to build up the helicity at large scales, we have to destroy magnetic helicity at small scales (Fig. 9).

Open boundary conditions would help to get rid of magnetic helicity (see Blackman \& Field 2000), which may well be an important factor in more realistic simulations (e.g., Glatzmaier \& Roberts 1995; Brandenburg et al. 1995). However, the present simulations are for closed boxes and yet they do show strong fields, so we need to understand whether and how they have been affected by this constraint.

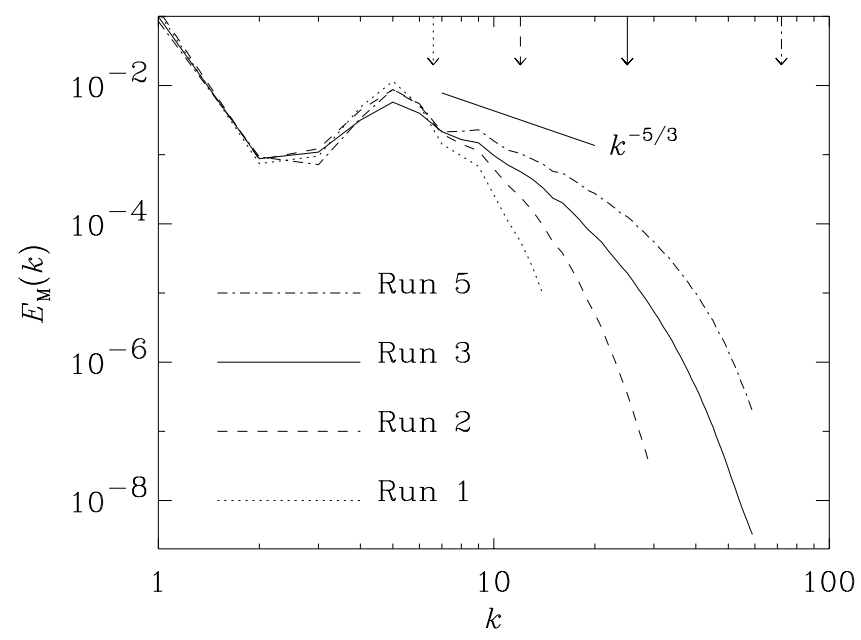

FIG. 19. - Comparison of time-averaged magnetic energy spectra for runs $1-3(t=600-1000)$ and run $5(t=1600)$. To compensate for different field strengths and to make the spectra overlap at large scales, two of the three spectra have been multiplied by a scaling factor $(\times 3.4$ for run $5, \times 2$ for run 2 , and $\times 5$ for run 1). There are signs of a gradual development of an inertial subrange for wavenumbers larger than the forcing scale. The $k^{-5 / 3}$ slope is shown for orientation. The dissipative magnetic cutoff wavenumbers, $k_{d}=\left\langle\boldsymbol{J}^{2} / \eta^{2}\right\rangle^{1 / 4}$, are indicated by arrows at the top. 
The rate at which magnetic helicity can change (see eq. [11]) is constrained by the Schwarz inequality,

$$
\left|\frac{d}{d t}\langle\boldsymbol{A} \cdot \boldsymbol{B}\rangle\right|=2 \eta|\langle\boldsymbol{J} \cdot \boldsymbol{B}\rangle| \leq 2 \eta J_{\mathrm{rms}} B_{\mathrm{rms}}
$$

(Berger 1984; Moffatt \& Proctor 1985). In forced systems it is common (albeit not necessary) that the energy $\left(B_{\mathrm{rms}}^{2}\right)$ and dissipation rate $\left(\eta J_{\mathrm{rms}}^{2}\right)$ are independent of the Reynolds number. However, this would imply that the rate of change of $\langle\boldsymbol{A} \cdot \boldsymbol{B}\rangle$ is limited resistively by a term proportional to $\eta^{1 / 2}$. In our simulations, $\langle\boldsymbol{J} \cdot \boldsymbol{B}\rangle /\left(\boldsymbol{J}_{\mathrm{rms}} \boldsymbol{B}_{\mathrm{rms}}\right)$ has a maximum of $\sim 0.25$ (see Table 1), so this limit does not seem to be saturated. Another estimate for the helicity dissipation is obtained by assuming that the magnetic spectrum has power-law behavior with $E_{M}(k) \sim k^{-n}$ for $k<k_{d}$. The value of $k_{\max }$ follows by assuming that the Joule dissipation is independent of $\eta$, which yields $k_{d} \sim \eta^{-1 /(3-n)}\left(\sim \eta^{-3 / 4}\right.$ for $n=5 / 3$ ). The helicity dissipation is then proportional to $k_{d}^{-1} \sim \eta^{1 /(3-n)}$, which always tends to zero for small values of $\eta$. For $n=5 / 3$ the magnetic helicity dissipation scales like $\eta^{3 / 4}$, which is faster than Berger's limit.

There is a related and even more important consequence of helicity conservation. In a steady state, $\langle\boldsymbol{A} \cdot \boldsymbol{B}\rangle$, which is gauge invariant and therefore physically meaningful, must be constant, and hence $\langle\boldsymbol{J} \cdot \boldsymbol{B}\rangle \rightarrow 0$. Thus, although we are going to generate a strong large-scale field with significant current helicity in the large scales, the net current helicity must actually vanish. This can only happen if there is an equal amount of small-scale current helicity of opposite sign, i.e.,

$$
\langle\boldsymbol{j} \cdot \boldsymbol{b}\rangle \approx-\langle\overline{\boldsymbol{J}} \cdot \overline{\boldsymbol{B}}\rangle \quad \text { (in the steady state) },
$$

where angular brackets denote volume averages, bars denote the large scales at $k=1$, and lowercase characters refer to contributions from all higher wavenumbers. The spectrum of the magnetic helicity is $k^{2}$ times steeper than that of the current helicity, so $\langle\boldsymbol{a} \cdot \boldsymbol{b}\rangle \approx\langle\boldsymbol{j} \cdot \boldsymbol{b}\rangle / k_{f}^{2}$, and therefore $|\langle\boldsymbol{a} \cdot \boldsymbol{b}\rangle| \ll|\langle\overline{\boldsymbol{A}} \cdot \overline{\boldsymbol{B}}\rangle|$.

In the following we estimate the evolution of the energy density of the large-scale field, $\overline{\boldsymbol{B}}^{2}$, using the fact that in the present simulations a large fraction of the magnetic energy is contained in the large-scale field (Fig. 7) and that magnetic helicity is strong (Fig. 8). This would be too ideal an assumption for astrophysical settings, but it is adequate for describing our present simulations. Thus, we may set

$$
k_{1}\langle\boldsymbol{A} \cdot \boldsymbol{B}\rangle \approx k_{1}\langle\overline{\boldsymbol{A}} \cdot \overline{\boldsymbol{B}}\rangle \approx-\left\langle\overline{\boldsymbol{B}}^{2}\right\rangle \approx \frac{\langle\overline{\boldsymbol{J}} \cdot \overline{\boldsymbol{B}}\rangle}{k_{1}} .
$$

For clarity we retain here the $k_{1}$ factors, even though they are equal to 1 . Using equation (11), this yields

$$
\frac{d}{d t}\left\langle\overline{\boldsymbol{B}}^{2}\right\rangle \approx-2 \eta k_{1}^{2}\left\langle\overline{\boldsymbol{B}}^{2}\right\rangle+2 \eta k_{1}|\langle\boldsymbol{j} \cdot \boldsymbol{b}\rangle|,
$$

which has the solution

$$
\left\langle\overline{\boldsymbol{B}}^{2}\right\rangle \approx 2 \eta k_{1} e^{-2 \eta k_{1}{ }^{2} t} \int_{t_{\mathrm{sat}}}^{t} e^{2 \eta k_{1} t^{\prime}}|\langle\boldsymbol{j} \cdot \boldsymbol{b}\rangle| d t^{\prime},
$$

which is expected to apply after the time of saturation, $t=$ $t_{\text {sat }}$, when $\langle\boldsymbol{j} \cdot \boldsymbol{b}\rangle$ is approximately constant, so

$$
\left\langle\overline{\boldsymbol{B}}^{2}\right\rangle \approx \frac{|\langle\boldsymbol{j} \cdot \boldsymbol{b}\rangle|}{k_{1}}\left[1-e^{-2 \eta k_{1}^{2}\left(t-t_{\mathrm{sat}}\right)}\right] .
$$

To a good approximation we may assume $\langle\boldsymbol{j} \cdot \boldsymbol{b}\rangle \approx$ $\rho_{0}\langle\boldsymbol{\omega} \cdot \boldsymbol{u}\rangle$. This means that the "residual helicity" (Pouquet et al. 1976), $\langle\boldsymbol{\omega} \cdot \boldsymbol{u}\rangle-\langle\boldsymbol{j} \cdot \boldsymbol{b}\rangle / \rho_{0}$, is small, which is indeed consistent with the present data and also with the results of Brandenburg \& Subramanian (2000), who used the ambipolar diffusion model of Subramanian (1999). The kinetic helicity can be approximated by $\langle\boldsymbol{\omega} \cdot \boldsymbol{u}\rangle \approx k_{f}\left\langle\boldsymbol{u}^{2}\right\rangle$, and so the final field strength, $B_{\text {fin }}$, is given by

$$
B_{\mathrm{fin}}^{2} \approx \frac{|\langle\boldsymbol{j} \cdot \boldsymbol{b}\rangle|}{k_{1}} \approx \rho_{0} \frac{|\langle\boldsymbol{\omega} \cdot \boldsymbol{u}\rangle|}{k_{1}} \approx \rho_{0}\left\langle\boldsymbol{u}^{2}\right\rangle \frac{k_{f}}{k_{1}} \equiv B_{\mathrm{eq}}^{2} \frac{k_{f}}{k_{1}} .
$$

For runs 1-3 and 5-7, the ratios of the actual values of $B_{\text {fin }}$ to those anticipated from equation (46) are $0.61,0.76,0.85$, $1.16,0.74$, and 0.36 . In the large- $R_{m}$ cases (runs 3 and 5) the ratio is close to 1 , thus confirming the assumption of small residual helicity in the saturated state.

The resistively limited growth of $\overline{\boldsymbol{B}}$ has immediate consequences for $\alpha$. The evolution equation for $\langle\overline{\boldsymbol{A}} \cdot \overline{\boldsymbol{B}}\rangle$ can be derived from equation (20),

$$
\frac{d}{d t}\langle\overline{\boldsymbol{A}} \cdot \overline{\boldsymbol{B}}\rangle=2 \alpha\left\langle\overline{\boldsymbol{B}}^{2}\right\rangle-2 \eta_{T}\langle\overline{\boldsymbol{J}} \cdot \overline{\boldsymbol{B}}\rangle .
$$

In the strongly helical case considered here the magnetic helicity of the large-scale field, $\langle\overline{\boldsymbol{A}} \cdot \overline{\boldsymbol{B}}\rangle$, is very nearly equal to $\langle\boldsymbol{A} \cdot \boldsymbol{B}\rangle$, so the right-hand sides of equations (11) and (47) must be approximately equal, which leads to

$$
|\alpha|-\eta_{T} k_{1} \approx \frac{\eta|\langle\boldsymbol{J} \cdot \boldsymbol{B}\rangle|}{\left\langle\overline{\boldsymbol{B}}^{2}\right\rangle} .
$$

In order to check this relation, we plot the two sides of equation (48) versus time (Fig. 20), where $\eta_{T}=\eta+\eta_{t}$ and $|\alpha|-\eta_{t} k_{1}$ has been obtained directly from $\mathscr{E}=\boldsymbol{u} \times \boldsymbol{B}$ assuming $\overline{\mathscr{E}}=\alpha \overline{\boldsymbol{B}}-\eta_{t} \overline{\boldsymbol{J}}$, which, for fully helical mean fields, leads to

$$
|\alpha|-\eta_{t} k_{1}=\frac{|\langle\overline{\mathscr{E}} \cdot \overline{\boldsymbol{B}}\rangle|}{\left\langle\overline{\boldsymbol{B}}^{2}\right\rangle} .
$$

(Note that here $\eta_{t}$ enters, not $\eta_{T}$. Recall also that in our case $\alpha<0$, so $\langle\overline{\mathscr{E}} \cdot \overline{\boldsymbol{B}}\rangle\langle 0$.) The condition (eq. [48]) on $\alpha$ reflects the fact that the growth of the large-scale field is limited by

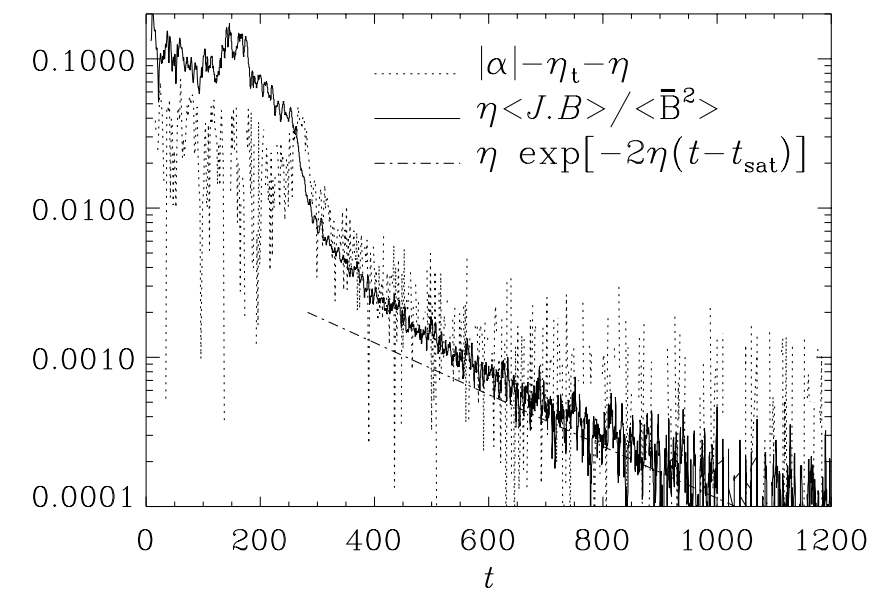

FIG. 20.-Comparison of $|\alpha|-\eta_{T} k_{1}$ with $\eta\langle\boldsymbol{J} \cdot \boldsymbol{B}\rangle /\left\langle\overline{\boldsymbol{B}}^{2}\right\rangle$ and $\eta \exp$ $\left[-2 \eta k_{1}^{2}\left(t-t_{\text {sat }}\right)\right]$. Note that $|\alpha|-\eta_{T} k_{1}$ follows very closely the resistively dominated limit. Notice also the relatively large noise level in $|\alpha|-\eta_{T}$ (run 3). 
the microscopic resistivity, as seen already from equation (45). Note, however, that this statement only applies to the present case of strong helicity and closed or periodic boxes.

There have been previous attempts to incorporate the conservation of helicity into the expression for $\alpha$, which led to a dynamical dependence of $\alpha$ on time and field strength (Kleeorin, Rogachevskii, \& Ruzmaikin 1995; Kleeorin et al. 2000). In the following we point out, however, that the helicity constraint, which leads to the prolonged saturation phase, is well described in terms of an $\alpha^{2}$ dynamo with resistively dominated quenching functions for both $\alpha$ and $\eta_{t}$, i.e.,

$$
\alpha=\frac{\alpha_{0}}{1+\alpha_{B} \overline{\boldsymbol{B}}^{2} / B_{\mathrm{eq}}^{2}}, \quad \eta_{t}=\frac{\eta_{t 0}}{1+\eta_{B} \overline{\boldsymbol{B}}^{2} / B_{\mathrm{eq}}^{2}},
$$

where $\alpha_{B}=\eta_{B}$ is assumed. Using the fact that the magnetic energy density of the mean field, $\overline{\boldsymbol{B}}^{2}$, is approximately uniform, we can write equation (20) in the form

$$
\frac{d}{d t}\left(\frac{1}{2} \overline{\boldsymbol{B}}^{2}\right)=\frac{\left|\alpha_{0}\right| k_{1}-\eta_{t 0} k_{1}^{2}}{1+\alpha_{B} \overline{\boldsymbol{B}}^{2} / \bar{B}_{\mathrm{eq}}^{2}} \overline{\boldsymbol{B}}^{2}-\eta k_{1}^{2} \overline{\boldsymbol{B}}^{2}
$$

There is a steady solution with

$$
\frac{\alpha_{B} \bar{B}^{2}}{B_{\mathrm{eq}}^{2}}=\frac{\lambda}{\eta k_{1}^{2}}
$$

where

$$
\lambda=\left|\alpha_{0}\right| k_{1}-\eta_{T 0} k_{1}^{2}
$$

is the kinematic growth rate of the dynamo. The timedependent equation (51) can be integrated to give the solution in the form

$$
\frac{\bar{B}^{2}}{\left(1-\overline{\boldsymbol{B}}^{2} / B_{\mathrm{fin}}^{2}\right)^{1+\lambda / \eta k_{1}{ }^{2}}}=B_{\mathrm{ini}}^{2} e^{2 \lambda t},
$$

where $B_{\text {ini }}$ is the initial field strength and $B_{\text {fin }}$ is the final field strength that is given by equation (52). The parameters $B_{\text {ini }}$ and $B_{\text {fin }}$ can be obtained from Figure 1 . The kinematic growth rate $\lambda$ is the same for small- and large-scale fields and hence can be taken from Table 1. We emphasize that there is excellent agreement between the results of the simulation and equation (54) (see Fig. 21).

Having obtained $\lambda$ and $B_{\text {fin }}$ from the simulation, we can use equation (52) to find for $\alpha_{B}$ and $\eta_{B}$ the result

$$
\alpha_{B}=\eta_{B}=\frac{\lambda}{\eta k_{1}^{2}}\left(\frac{B_{\mathrm{eq}}}{B_{\mathrm{fin}}}\right)^{2} .
$$

Note that $\alpha_{B}$ and $\eta_{B}$ are proportional to $1 / \eta$, which implies $R_{m}$-dependent $\alpha$ and $\eta_{t}$ quenchings. The results for $\alpha_{B}=\eta_{B}$ are summarized in Table 3 for different runs.

To a good approximation $B_{\mathrm{fin}}^{2} / B_{\mathrm{eq}}^{2} \approx k_{f} / k_{1}$ (see eq. [46]), so we expect $\alpha_{B} \approx \lambda /\left(\eta k_{1} k_{f}\right)$. Since $\lambda \approx 0.3 u_{\text {rms }} / \ell_{f}$, we have

$$
\alpha_{B} \approx \frac{0.3}{(2 \pi)^{2}} \frac{u_{\mathrm{rms}} L}{\eta} \approx 0.01 R_{m, \text { lin }} .
$$

Thus, $\alpha_{B}$ should scale with the magnetic Reynolds number based on the box scale, not the forcing scale. The difference is particularly evident when comparing with run 6 , where the forcing wavenumber is 6 times larger than in the other runs. For comparison we list in Table 3 also $R_{m, \operatorname{lin}} / 100$ and $R_{m, \text { forc }} / 20$. The agreement between equations (55) and (56) is generally good, except for run 7 , where $B_{\text {fin }}$ is smaller than expected from equation (46). This was the run with a small value of $v / \eta$ and small $R_{m}$.

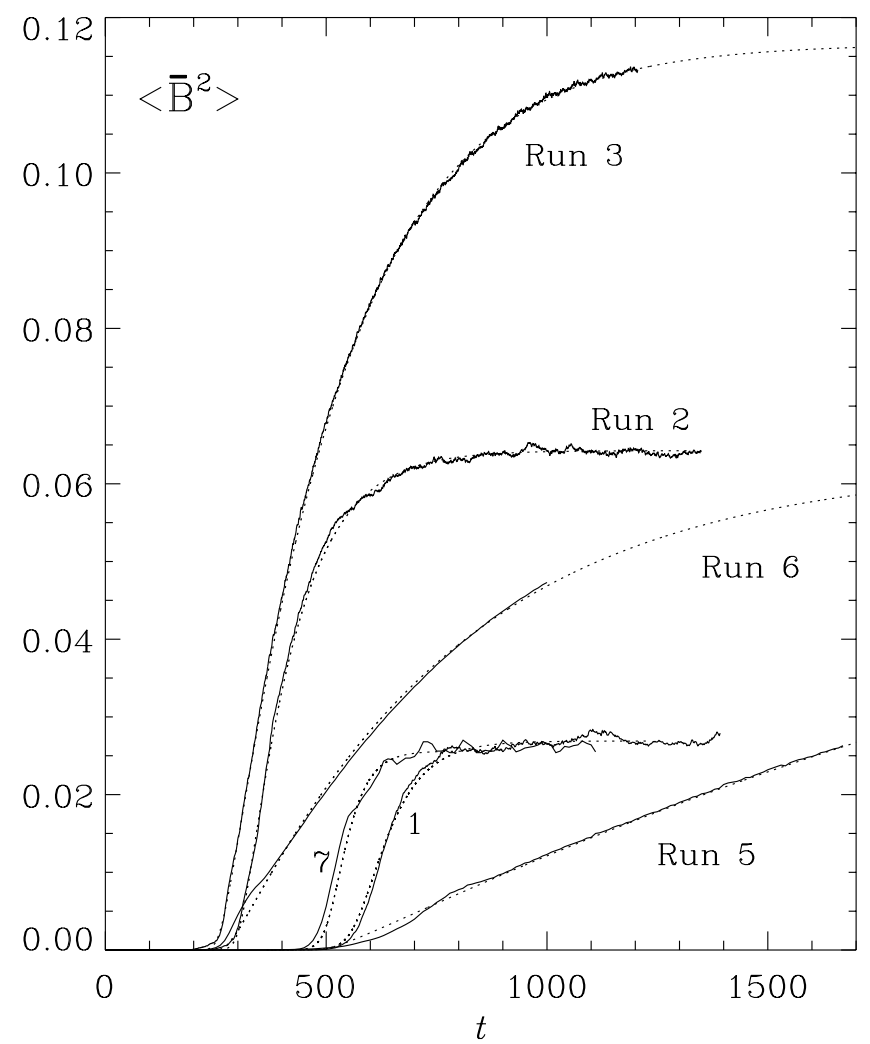

FIG. 21.-Evolution of $\left\langle\overline{\boldsymbol{B}}^{2}\right\rangle$ for runs 1-3 and 5-7 (solid lines), compared with the solution (eq. [54]) of the mean field dynamo equations using resistively dominated $\alpha$ and $\eta_{t}$ quenchings (dotted lines).

In Table 3 we also give for completeness the values of $B_{\text {ini. }}$. Together with the values of $\eta$ and $\lambda$ in Table 1 all the data entering equation (54) are now specified. It should be mentioned that the value of $B_{\text {ini }}$ is obtained from a fit and is only roughly comparable to the actual seed magnetic field in the simulation, where different initial structures are possible.

For weak fields equation (54) gives the usual exponential growth, $|\overline{\boldsymbol{B}}|=B_{\text {ini }} \exp (\lambda t)$. For strong fields we recover equation (45) with $t_{\mathrm{sat}}=\lambda^{-1} \ln \left(B_{\mathrm{fin}} / B_{\mathrm{ini}}\right)$ in the limit $\lambda \gg$ $\eta k_{1}^{2}$.

\subsection{Large Magnetic Prandtl Numbers}

The magnetic helicity constraint becomes more important as the magnetic Reynolds number is increased. So far we have mainly considered the case in which $v / \eta=1$. In the Sun and many other astrophysical bodies, $v / \eta \ll 1$, but in the Galaxy $v / \eta \gg 1$ (e.g., Kulsrud \& Andersen 1992). This

TABLE 3

COEFFICIENTS FOR THE $\alpha$ QUENCHING EXPRESSION OBTAINED FROM Equation (55) for Runs with Different Values of Magnetic REYNOLDS NUMBER

\begin{tabular}{lccccc}
\hline \hline Run & $R_{m, \text { lin }} / 100$ & $R_{m, \text { forc }} / 20$ & $\alpha_{B}$ & $B_{\text {fin }}$ & $B_{\text {ini }}$ \\
\hline $1 \ldots \ldots$ & 1 & 1 & 1.4 & 0.16 & $3 \times 10^{-8}$ \\
$2 \ldots \ldots$ & 3 & 3 & 3.9 & 0.25 & $3 \times 10^{-9}$ \\
$3 \ldots \ldots$ & 9 & 9 & 9.3 & 0.34 & $2 \times 10^{-9}$ \\
$5 \ldots \ldots$ & 35 & 35 & 30 & 0.27 & $4 \times 10^{-11}$ \\
$6 \ldots \ldots$ & 5 & 1 & 4.6 & 0.25 & $4 \times 10^{-11}$ \\
$7 \ldots \ldots$ & 1 & 1 & 2.8 & 0.16 & $1 \times 10^{-9}$ \\
\hline
\end{tabular}

NoTE.- Note the rough agreement between the values of $R_{m, \text { lin }} / 100$ and $\alpha_{B}$. 

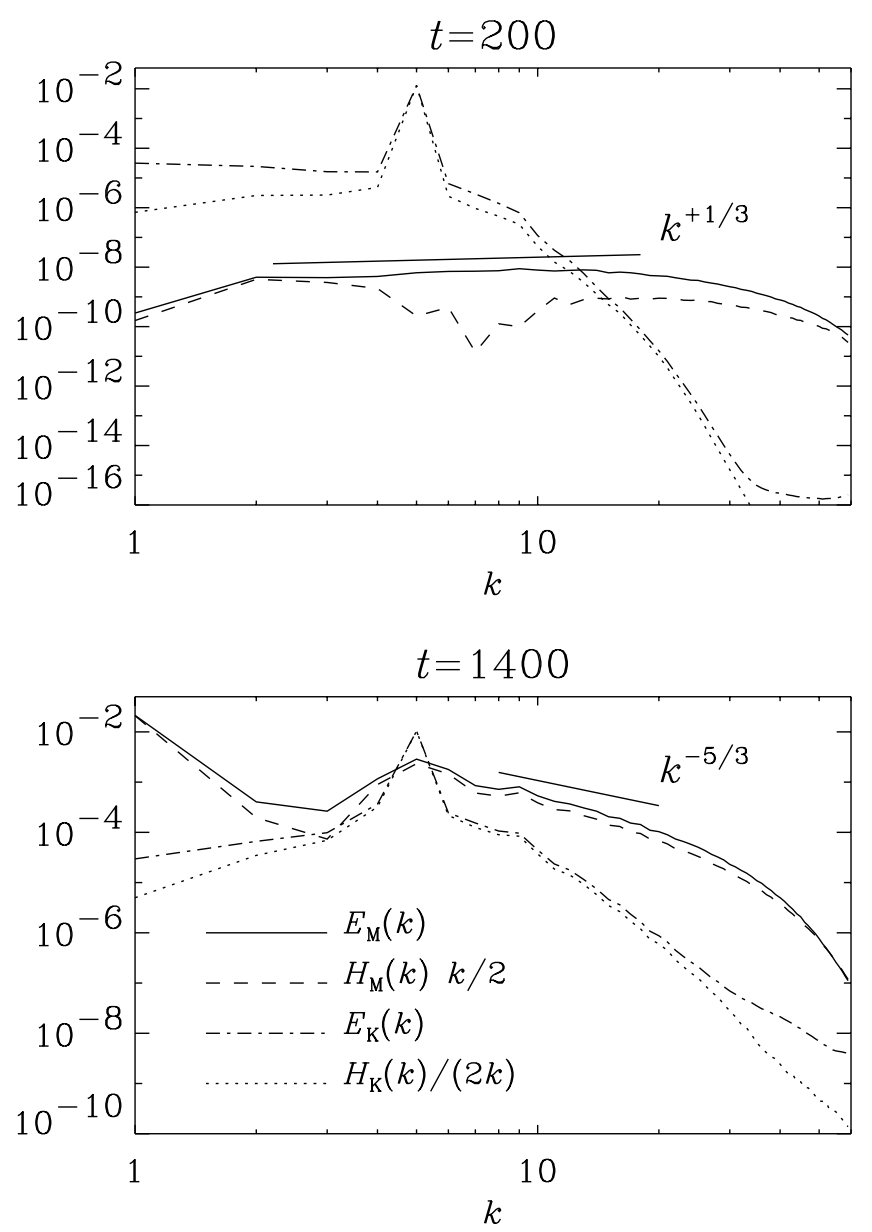

FIG. 22.- Spectra of magnetic and kinetic energy and helicity for a large magnetic Prandtl number at early and late times; $v=0.02$, $\eta=2 \times 10^{-4}$.

may lead to a magnetic energy spectrum peaking at small scales (Kulsrud \& Andersen 1992). However, although viscous damping will dissipate energy at small scales (Chandran 1998; Kinney et al. 1998), there is some recent evidence that the inverse cascade may no longer operate

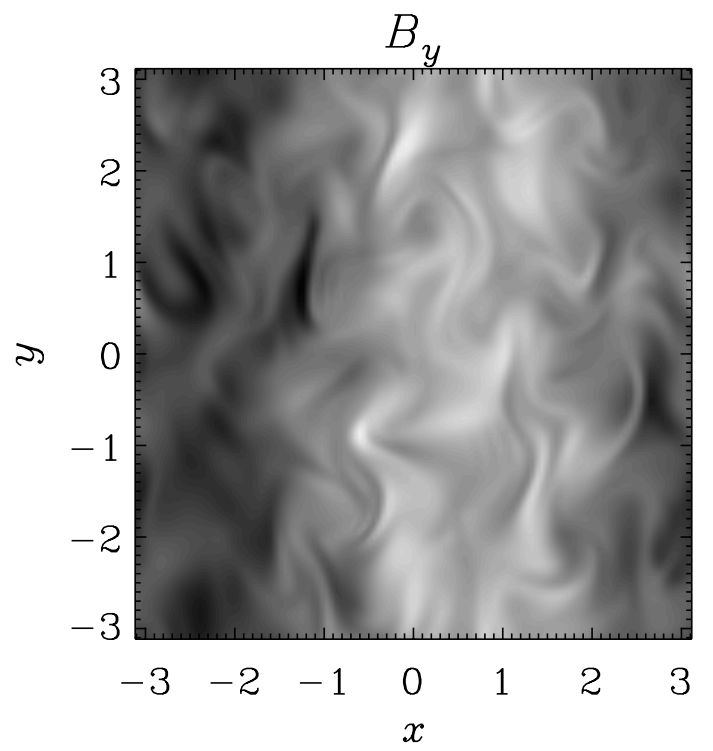

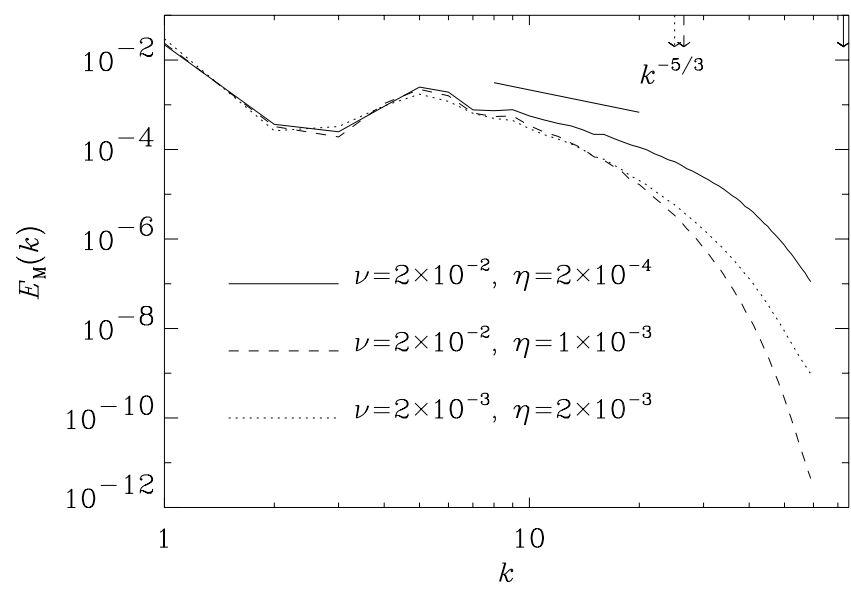

FIG. 23.-Comparison of the magnetic energy spectra for runs 3 , 4, and 5. The magnetic cutoff wavenumbers are 25,26 , and 72 , as indicated by arrows at the top.

(Maron 2000). These results have been obtained in the absence of net helicity. It will therefore be interesting to see whether in the presence of net helicity an inverse cascade is still possible when $v / \eta \gg 1$.

In a preliminary attempt to clarify this question we have carried out simulations for $v / \eta=20$ (mainly by increasing the viscosity to $v=0.02$; run 4) and $v / \eta=100$ (where the magnetic diffusivity was lowered to $\eta=2 \times 10^{-4}$; run 5). The viscous cutoff wavenumber is then around 5, i.e., at the forcing scale, and the magnetic cutoff wavenumbers are 25 and 72, respectively. The resolution for run 5 may be insufficient, and discretization errors must play a role at small scales, but the images of the current density look reasonable (see below), and the evolution of the large-scale field is also in agreement with expectations $(\S 3.6)$.

As seen from Figure 22, the magnetic energy spectrum does indeed peak at large wavenumbers initially, although the magnetic energy spectrum does not scale like $k^{3 / 2}$ (Kulsrud \& Andersen 1992). Instead, the spectrum is close to $k^{1 / 3}$, which was also found during the kinematic stage of convective dynamos (Brandenburg et al. 1996). However, this result is inconclusive because it could be an artifact of

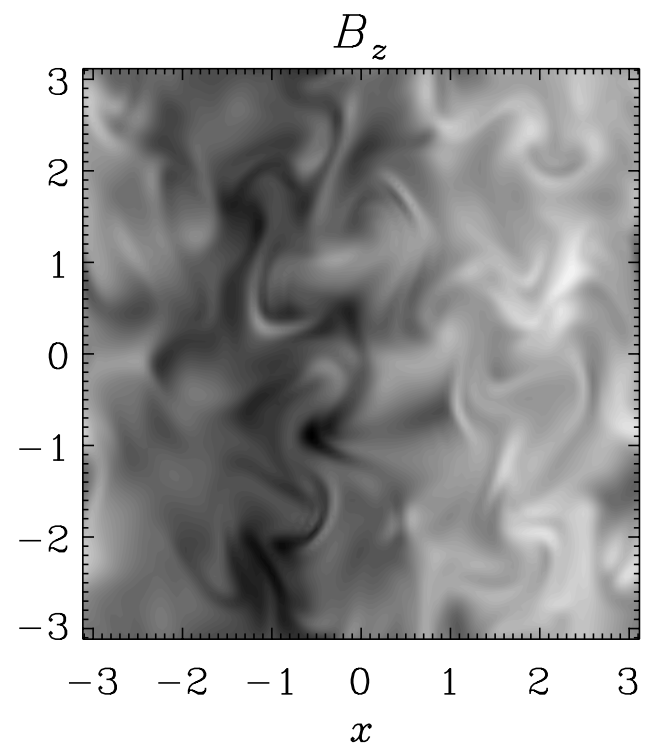

FIG. 24. - Images of the $B_{y}$ and $B_{z}$ components of the magnetic field in an arbitrarily chosen $x-y$ plane. Run 5, $t=1600$. 

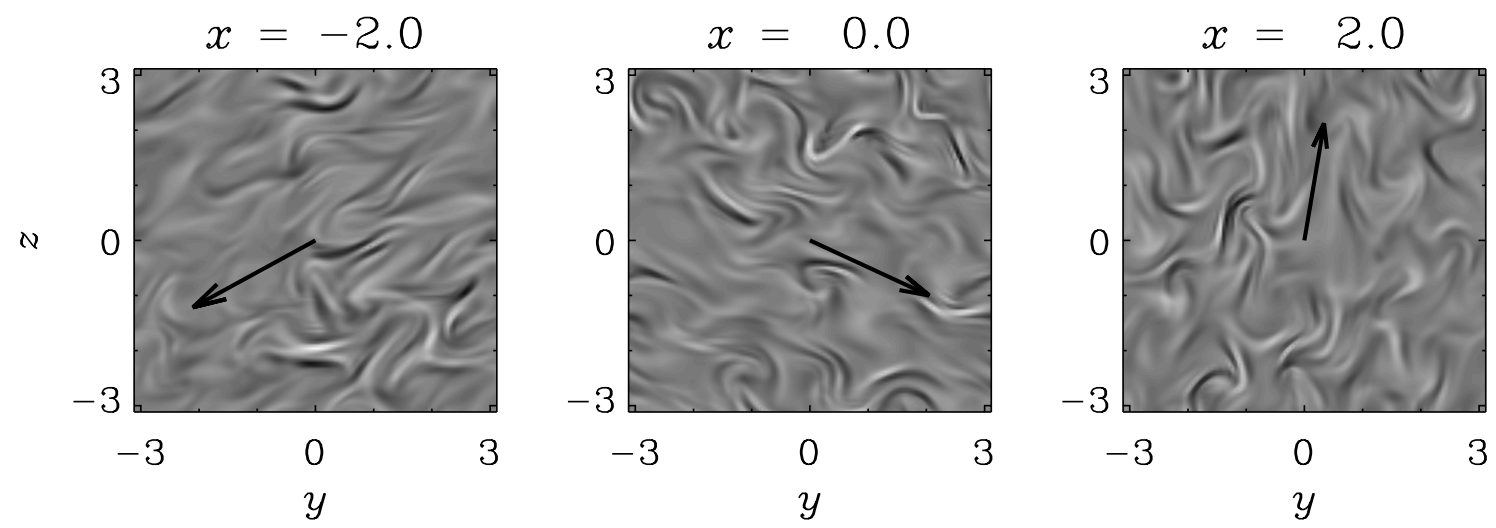

FIG. 25.-Images of the current density, $J_{x}$, in the plane of the large-scale magnetic field. The field direction is shown as a vector. Run $5, t=1600$.

the lack of an inertial range in this run. In any case, at large wavenumbers the magnetic energy exceeds the kinetic energy, although at later times the kinetic energy is increased somewhat by magnetic forces. Especially at later times the magnetic energy is no longer dominated by small scales and the spectrum falls off more like $k^{-5 / 3}$. The convergence to this power law is evident when comparing runs 3, 4, and 5 (Fig. 23). Most importantly, there are now clear signs of an inverse cascade (see Fig. 24).

In these runs with large magnetic Prandtl number the current density shows strong filamentary structures that tend to be aligned with the local magnetic field direction, as seen in Figure 25. The resulting anisotropy affects particularly the small scales (Goldreich \& Sridhar 1997; Maron 2000). Note that this type of anisotropy cannot be captured by closure models (e.g., Pouquet et al. 1976).

The main shortcoming of the present large Prandtl number calculations is that the viscous dissipation cutoff wavenumber is so small that it lies in the range of the forcing scale, so no inertial range in the kinetic energy is possible. At the same time, of course, the range of scales available to the magnetic field is still not large enough to establish a $k^{3 / 2}$ scaling at early times.

\section{CONCLUSIONS}

The main conclusion to be drawn from this work is that in the presence of net magnetic helicity there is a gradual buildup of a nearly force-free magnetic field at the largest possible scale of the system. In our periodic calculations this corresponds to a sinusoidal one-dimensional Beltrami field, e.g., $\overline{\boldsymbol{B}} \sim(\cos z, \sin z, 0)$, which is, of course, locally strongly distorted by the turbulence. Nevertheless, the presence of the large-scale field is clearly seen without averaging (Fig. 4). We emphasize that this result is numerically robust: the relative dominance of magnetic energy at the smallest wavenumber is independent of resolution (Fig. 19) and independent of the degree of scale separation (Fig. 17). Thus, the effect is seen equally well at resolutions ranging from $30^{3}$ to $120^{3}$ mesh points and at forcing wavenumbers ranging from 5 to 30 . However, the time it takes to establish such largescale fields increases with the ohmic diffusion time. We also note that the results are not very sensitive to the choice of the forcing function: a forcing function that is nearly $\delta$-correlated in space, but still strongly helical, yields very similar results. In the absence of net helicity, however, no large-scale field is generated. Likewise, if the forcing is made nonhelical, the large-scale field disappears.
An important property of the turbulence is that once the large-scale field is established, it can suppress magnetic energy on scales smaller than the largest one. This leads to something like a "self-cleaning" process. This is also seen in histograms of the magnetic field, which are, for the present simulations, more nearly Gaussian (with one hump perpendicular to the field and two in the direction of the field). This is similar to other simulations with large-scale dynamo action (see Brandenburg et al. 1995) but very different from simulations of small-scale dynamo action where the histograms of the field components show stretched exponentials (Brandenburg et al. 1996), which can also be seen in the present simulations, but only at early times.

Our simulations show that most of the energy input to the large-scale field comes from small scales. This type of nonlocal spectral energy transfer is suggestive of an $\alpha$-effect that could be responsible for the field generation, rather than a local inverse cascade, which transports energy from $k=2$ to $k=1$, for example. Although a local inverse cascade seems to occur at early times, i.e., before the magnetic field is fully established, once the field is strong the magnetic energy at $k=2$ is actually cascaded to $k=3$ and/or transferred to kinetic energy, both of which are probably important for the "self-cleaning" process. A sketch of the anticipated energy transfer properties is given in Figure 26.

We point out that the present simulations must not be regarded as local in the sense of representing only a small

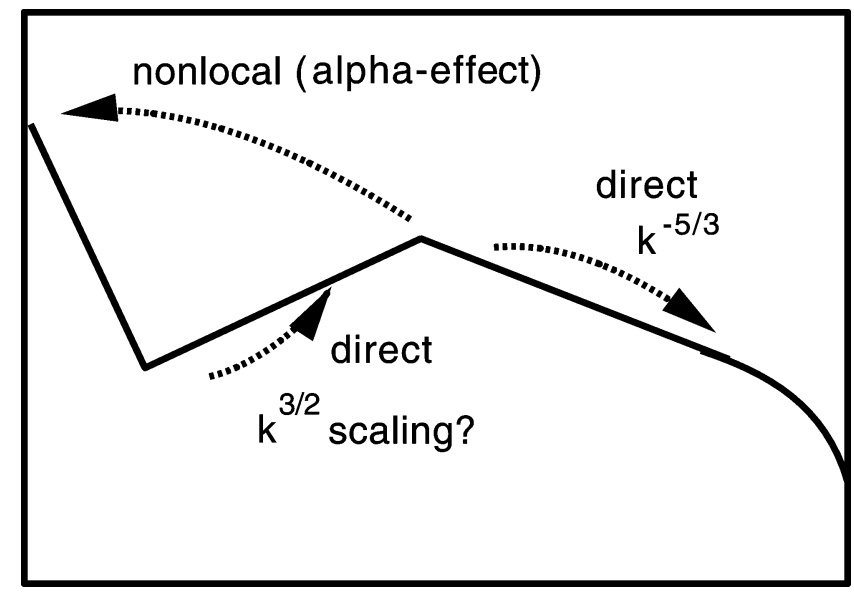

FIG. 26.- Sketch illustrating direct and inverse cascade processes in helical MHD turbulence. 
chunk of a larger system because the field structure depends crucially on the size of the box. Instead, they should be viewed as global within the geometry considered. With other boundary conditions or in different geometries the shape of the large-scale field will be different. In the case of a sphere, for example, no perfectly force-free field is possible, but the field may be nearly force free. An example may be the field obtained in hydromagnetic calculations with $\alpha$ effect (Proctor 1977), where field saturation occurs through the Lorentz force of the large-scale field. In these calculations the magnetic saturation field strength is relatively large, which reflects the fact that the field is indeed nearly force free.

It should be emphasized that the overall growth of the large-scale field and the saturation phase of the dynamo are well described by a simple $\alpha^{2}$ dynamo with $\alpha$ and $\eta_{t}$ coefficients that are quenched in an $R_{m}$-dependent fashion (see eqs. [50] and [55]). The reason such a dynamo can still saturate is because of the presence of microscopic diffusion, and it is this that causes the saturation to happen so slowly. The excellent agreement in the evolution toward saturation between both the simulation and the mean field model is an indication that the simple quadratic quenching formula is actually correct. For example, a cubic nonlinearity (Moffatt 1972; Rüdiger 1974) would lead to different behavior and would not have the correct resistive relaxation asymptotics consistent with helicity conservation (Brandenburg 2000).

The slow resistive field evolution past equipartition has become particularly clear in run 5 , where the final selection of the large-scale field structure occurred rather late (after $t \approx 1200$, corresponding to about 100 turnover times; Fig. 6). By contrast, in run 3, where the magnetic Reynolds number was about 6 times smaller, the large-scale field was fully developed by the time $t \approx 400$, corresponding to about 50 turnover times. In stars the typical magnetic Reynolds numbers are at least another 6 orders of magnitude larger than in run 5 , so a large-scale field, if generated by an $\alpha$ effect, would require $\sim 10^{8}$ turnover times or $\sim 3 \times 10^{6} \mathrm{yr}$ (assuming a turnover time of 10 days). In the case of the Sun this estimate would be reduced by another factor of 100 (Brandenburg et al. 2000) because differential rotation con- tributes to nonhelical field generation, so the resulting fields are only partially subject to the helicity constraint. Since even the youngest protostars are older than $3 \times 10^{4}$, the $\alpha-\omega$ dynamo may still be responsible for field generation in these bodies. For galaxies, on the other hand, the magnetic Reynolds numbers are by another 7 orders of magnitude larger than in stars, making here the case for an $\alpha-\omega$ dynamo doubtful, unless the microscopic resistivity is enhanced during reconnection (see Ji et al. 1998 for anomalous resistivities in a laboratory reconnection experiment).

There is now also some evidence that in oscillatory dynamos of $\alpha-\omega$ type the cycle period is not strongly affected by the helicity timescale constraint (Brandenburg et al. 2000). This could be related to the fact that with shear the large-scale field is no longer fully force free and that in that case the turbulent magnetic diffusivity is only partially suppressed (Gruzinov \& Diamond 1996). However, the case for $\alpha-\omega$ dynamo action in stars, galaxies, or accretion discs is by no means settled. First of all, proposals have been made for nonhelical large-scale dynamo action (Vishniac \& Cho 2000; Zheligovsky, Podvigina, \& Frisch 2000), which may avoid the problems that $\alpha-\omega$ dynamos have. Secondly, real astrophysical bodies do have open boundaries and may get rid of small-scale helicity rather rapidly (Berger \& Ruzmaikin 2000). Indications are, however, that open boundaries also produce significant losses at large scales, which lowers the overall dynamo efficiency (see Brandenburg \& Dobler 2000).

I thank the participants of the Astrophysical Turbulence Program at the Institute for Theoretical Physics at the University of California, Santa Barbara, for the many valuable discussions without which this work would not have been possible. I am grateful for the referees' criticisms, which lead to considerable improvements in the presentation of the results. This work was supported by the National Science Foundation through grant PHY94-07194 and PPARC through grant PPA/G/S/1997/00284. Use of the PPARCsupported supercomputers in St. Andrews and Leicester is acknowledged.

\section{REFERENCES}

Balsara, D., \& Pouquet, A. 1999, Phys. Plasmas, 6, 89

Berger, M. A. 1984, Geophys. Astrophys. Fluid Dyn., 30, 79

Berger, M. A., \& Ruzmaikin, A. 2000, J. Geophys. Res., 105, 10481

Biferale, L., \& Kerr, R. M. 1995, Phys. Rev. E, 52, 6113

Blackman, E. G., \& Chou, T. 1997, ApJ, 489, L95

Blackman, E. G., \& Field, G. F. 2000, ApJ, 534, 984

Brandenburg, A. 2000, in IAU Symp. 203, Recent Insights into the Physics of the Sun and Heliosphere, ed. P. Brekke, B. Fleck, \& J. B. Gurman, in press (astro-ph/0011579)

Brandenburg, A., Bigazzi, A., \& Subramanian, K. 2001, MNRAS, in press

Brandenburg, A., \& Dobler, W. 2001, A\&A, 369, 329

Brandenburg, A., \& Donner, K. J. 1997, MNRAS, 288, L29

Brandenburg, A., Jennings, R. L., Nordlund, A., Rieutord, M., Stein, R. F., \& Tuominen, I. 1996, J. Fluid Mech., 306, 325

Brandenburg, A., Nordlund, A., Pulkkinen, P., Stein, R. F., \& Tuominen, I. 1990, A\&A, 232, 277

Brandenburg, A., Nordlund, Å., Stein, R. F., \& Torkelsson, U. 1995, ApJ, 446,741

Brandenburg, A., \& Sokoloff, D. 2000, Geophys. Astrophys. Fluid Dyn., submitted

Brandenburg, A., \& Subramanian, K. 2000, A\&A, 361, L33

Cattaneo, F., \& Hughes, D. W. 1996, Phys. Rev. E, 54, R4532

Cattaneo, F., \& Vainshtein, S. I. 1991, ApJ, 376, L21

Chandran, B. D. G. 1998, ApJ, 492, 179

Field, G. B., Blackman, E. G., \& Chou, H. 1999, ApJ, 513, 638

Frisch, U., Pouquet, A., Léorat, J., \& Mazure, A. 1975, J. Fluid Mech., 68, 769
Galanti, B., Gilbert, A. D., \& Sulem, P.-L. 1990, in Topological Fluid Mechanics, ed. H. K. Moffatt \& A. Tsinober (Cambridge: Cambridge Univ. Press), 138

Galanti, B., Sulem, P.-L., \& Gilbert, A. D. 1991, Physica, 47, 416

Glatzmaier, G. A., \& Roberts, P. H. 1995, Nature, 377, 203

Goldreich, P., \& Sridhar, S. 1997, ApJ, 485, 680

Gruzinov, A. V., \& Diamond, P. H. 1996, Phys. Plasmas, 3, 1853

Ji, H. 1999, Phys. Rev. Lett., 83, 3198

Ji, H., Yamada, M., Hsu, S., \& Kulsrud, R. 1998, Phys. Rev. Lett., 80, 3256

Kazantsev, A. P. 1968, J. Exp. Theor. Phys., 26, 1031

Kinney, R. M., Chandran, B. D. G., Cowley, S. C., \& McWilliams, J. C. 1998, Am. Astron. Soc., 192, 66.18

Kleeorin, N. I., Moss, D., Rogachevskii, I., \& Sokoloff, D. 2000, A\&A, 361, L5

Kleeorin, N. I., Rogachevskii, I., \& Ruzmaikin, A. 1995, A\&A, 297, 159

Krause, F., \& Rädler, K.-H. 1980, Mean Field Magnetohydrodynamics and Dynamo Theory (Berlin: Akademie)

Kulsrud, R. M., \& Anderson, S. W. 1992, ApJ, 396, 606

Maron, J. L. 2000, Ph.D. thesis, Caltech

Meneguzzi, M., Frisch, U., \& Pouquet, A. 1981, Phys. Rev. Lett., 47, 1060

Moffatt, H. K. 1972, J. Fluid Mech., 53, 385

1978, Magnetic Field Generation in Electrically Conducting

Fluids (Cambridge: Cambridge Univ. Press)

Moffatt, H. K., \& Proctor, M. R. E. 1985, J. Fluid Mech., 154, 493

Parker, E. N. 1979, Cosmical Magnetic Fields (Oxford: Clarendon Press)

Pouquet, A., Frisch, U., \& Léorat, J. 1976, J. Fluid Mech., 77, 321

Proctor, M. R. E. 1977, Astron. Nachr., 80, 769

Rüdiger, G. 1974, Astron. Nachr., 295, 275 
Seehafer, N. 1996, Phys. Rev. E, 53, 1283

Subramanian, K. 1999, Phys. Rev. Lett., 83, 2957

Tao, L., Cattaneo, F., \& Vainshtein, S. I. 1993, in Solar and Planetary Dynamos, ed. M. R. E. Proctor, P. C. Matthews, \& A. M. Rucklidge (Cambridge: Cambridge Univ. Press), 303

Vainshtein, S. I., \& Cattaneo, F. 1992, ApJ, 393, 165

Vainshtein, S. I., Tao, L., Cattaneo, F., \& Rosner, R. 1993, in Solar and Planetary Dynamos, ed. M. R. E. Proctor, P. C. Matthews, \& A. M. Rucklidge (Cambridge: Cambridge Univ. Press), 311
Vishniac, E. T., \& Cho, J. 2000, ApJ, in press (astro-ph/0010373) Zheligovsky, V. A., Podvigina, O. M., \& Frisch, U. 2000, Geophys. Astrophys. Fluid Dyn., submitted

Ziegler, U., \& Rüdiger, G. 2000, A\&A, 356, 1141 